\title{
Systoles and minimal surfaces in 3-manifolds with boundary
}

\author{
Eduardo Rosinato Longa
}

THESIS SUBMITTED

TO THE

Institute of MAthematics AND StATistics

OF THE

University of SÃo PAUlo

IN

PARTIAL FULFILLMENT

$\mathrm{OF}$

THE REQUIREMENTS

FOR

OBTAINING THE DEGREE

OF

DOCTOR OF SCIENCE

Programme: Mathematics

Advisor: Professor Paolo Piccione

During the development of this project, the author received a CAPES/FAPESP fellowship

São Paulo, March 2021 


\section{Systoles and minimal surfaces in 3-manifolds with boundary}

This is the final version of the thesis corrected and publically defended by Eduardo Rosinato Longa on the 16th of March 2021 and approved by the Examining Committee. A copy or the original version is available at Instituto de Matemática e Estatística, Universidade de São Paulo.

Examining Committee:

- Professor Paolo Piccione (advisor) - Universidade de São Paulo

- Professor Marcos Martins Alexandrino da Silva - Universidade de São Paulo

- Professor Ruy Tojeiro de Figueiredo Junior - Universidade de São Paulo

- Professor Jaime Bruck Ripoll - Universidade Federal do Rio Grande do Sul

- Assistant Professor Lucas Coelho Ambrozio - Instituto de Matemática Pura e Aplicada 
Aos meus pais. 


\section{Acknowledgements}

Renda-se, como eu me rendi.

Mergulhe no que você não conhece como eu mergulhei.

Não se preocupe em entender, viver ultrapassa qualquer entendimento.

Clarice Lispector

Firstly, I thank my parents for their constant support and encouragement, and for turning the possibility of moving to São Paulo into reality. Other people were also very important in this long journey, especially my advisor Paolo Piccione, with whom I had very fruitful mathematical conversations and who presented me the fantastic world of board games, and Lucas Ambrozio, who has given me valuable advice and with whom I had clarifying discussions concerning the results of this thesis.

In 2019 I had the chance to make two life-changing trips. The first one was to New York, with Paolo. I thank Renato Bettiol for his hospitality and for the great time we had there. The second one was to participate in a Doc-course on Geometric Analysis in Spain. I express my gratitude to Paolo and Álvaro Ramos for encouraging me to register for this course. The activities were held at Sevilla, Granada and Cádiz. I thank all the universities in which I had the chance to work, and all the wonderful friends I made there.

Additionally, Luísa Borsato was fundamental in the early moments in São Paulo; we supported each other to overcome the initial dificulities that naturally arise for people that move. I also thank all the friendships that were established in the new city, especially those with Leonardo Cavenaghi, Adriana Nicoli and Jackeline Conrado. We shared incredible moments together, including an amazing (in a broad sense) algebra course, interesting geometry seminaries and long discussions about a variety of topics. Furthermore, I thank Izabella Freitas and Jackeline Conrado (once more) for the figures in this work. Finally, I would like to thank CAPES and FAPESP for the financial suport provided during the period of the $\mathrm{PhD}$, from march 2018 to march 2021: grant 2017/22704-0, São Paulo Research Foundation (FAPESP). 


\section{Resumo}

Longa, E. R., Systoles and minimal surfaces in 3-manifolds with boundary. 2021. Tese (Doutorado) - Instituto de Matemática e Estatística, Universidade de São Paulo, São Paulo, 2021.

Este trabalho tem dois objetivos. Primeiramente, provamos a existência de uma folheação local em torno de superfícies capilares infinitesimalmente rígidas. Usamos então este fato para mostrar um resultado de rigidez para superfícies capilares infinitesimalmente rígidas em algumas variedades Riemannianas de dimensão 3 com bordo convexo em média. Também derivamos cotas para o gênero, número de componentes do bordo e área de qualquer superfície capilar mínima compacta com dois lados e índice baixo sob certas suposições na curvatura do ambiente e na de seu bordo. Em segundo lugar, provamos algumas desigualdades sistólicas ótimas para variedades compactas de dimensão 3 com bordo. Elas relacionam as sístoles homológicas (relativas) da variedade com sua curvatura escalar e curvatura média do bordo. No caso de igualdade, o recobrimento universal da variedade é isométrico a um cilindro sobre um disco de curvatura constante e não negativa.

Palavras-chave: superfícies mínimas, sístole, rigidez, curvatura escalar, geometria Riemanniana. 


\section{Abstract}

Longa, E. R., Systoles and minimal surfaces in 3-manifolds with boundary. 2021. Tese (Doutorado) - Instituto de Matemática e Estatística, Universidade de São Paulo, São Paulo, 2021.

The aim if this work is twofold. Firstly, we prove the existence of a local foliation around compact infinitesimally rigid capillary surfaces. We then use this fact to show a rigidity result for infinitesimally rigid capillary surfaces in some Riemannian 3-manifolds with mean convex boundary. We also derive bounds on the genus, number of boundary components and area of any compact two-sided capillary minimal surface with low index under certain assumptions on the curvature of the ambient manifold and of its boundary. Secondly, we prove some sharp systolic inequalities for compact 3-manifolds with boundary. They relate the (relative) homological systoles of the manifold to its scalar curvature and mean curvature of the boundary. In the equality case, the universal cover of the manifold is isometric to a cylinder over a disc of nonnegative constant curvature.

Keywords: minimal surfaces, systole, rigidity, scalar curvature, Riemannian geometry. 


\section{Contents}

List of Figures $\quad$ xiii

1 Introduction $\quad 1$

2 Geometric preliminaries $\quad 9$

2.1 Riemannian Geometry . . . . . . . . . . . . . . . . . . . . . . . . . 9

2.2 Algebraic Topology . . . . . . . . . . . . . . . . . . . . . . 10

3 Capillary surfaces $\quad 13$

3.1 Preliminaries: variational problem and stability . . . . . . . . . . . . . . . . 13

3.2 Results . . . . . . . . . . . . . . . . . . . . . . 16

$\begin{array}{lll}4 & \text { Systolic inequalities } & 27\end{array}$

4.1 Homological systoles . . . . . . . . . . . . . . . . . . . . . . . . 27

4.2 Results . . . . . . . . . . . . . . . . . . . . . . 29

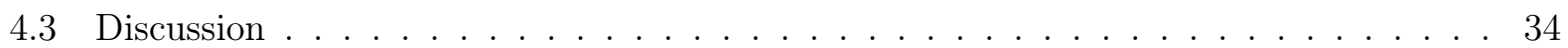

$\begin{array}{ll}\text { References } & 39\end{array}$ 


\section{List of Figures}

1.1 Two minimal surfaces with the same boundary. . . . . . . . . . . . . . . 2

1.2 An arrangement of 3 planes in $\mathbb{R}^{3}$ seen from a plane orthogonal to the line $\ell$. . . . 4

1.3 The systole is realised be the red curve in this torus. . . . . . . . . . . . . . 5

1.4 The disc $D$ realises the quantity $\mathcal{A}(M, g)$, whereas $D^{\prime}$ realises $\operatorname{sys}_{2}(M, \partial M) \ldots \ldots$

3.1 The four fundamental vector fields in a capillary minimal surface in a cone. . . . . . 15

4.1 Two minimal surfaces with the same boundary. . . . . . . . . . . . . . 35 


\section{Chapter 1}

\section{Introduction}

Whereas topologists are interested in properties of spaces that are invariant under continuous deformations like stretching, twisting and bending (but not tearing or gluing), geometers give more attention to properties that can be measured, like distances, lengths, areas and curvature. Most of these measurements can only be made in spaces equipped with a Riemannian metric. This is the realm of Riemannian Geometry, which is the general area this thesis fits into.

The objects studied by Riemannian Geometry are called Riemannian manifolds, which are differentiable manifolds on which we are allowed to perform measurements such as the ones mentioned above. To study a manifold $M$, it is often useful to understant what are the possible interesting submanifolds $M$ supports. Of course, we need to specify what interesting means in this context. For the purposes of this work, the relevant submanifolds are the minimal submanifolds (other possibilities include CMC, Weingarten or totally geodesic submanifolds, for instance).

Throughout the text, we always consider minimal surfaces lying in 3-dimensional ambient manifolds. They arise naturally as the shape we see after we dip a wire frame into a soap solution. The soap film that is formed when we take the frame out minimises the energy among all surfaces having this frame as a boundary. This energy is the potential energy resulted from the intermolecular force. Assuming the thickness of the soap film is uniform, the energy is proportional to the area of the surface. As a result, the soap films assumes a configuration that minimises area.

The problem of minimising the area of surfaces that have a fixed boundary - known as Plateau problem — was first studied by Lagrange [Lag60] in 1760, when he discovered that the problem was governed by a partial differential equation. Later, in 1776, Meusnier [Meu85] showed that areaminimising surfaces have vanishing mean curvature. Ever since, the theory of minimal surfaces ramified in many different branches, including the study of these surfaces in other ambient geometries (positive and negative curvature, for example), high dimension and codimension cases, and the classical theory in $\mathbb{R}^{3}$.

As mentioned above, area-minimising surfaces are minimal surfaces, but the converse might not hold. For instance, let $C_{1}$ and $C_{2}$ be two circles of the same radius that lie on parallel planes and whose centers determine a line that is orthogonal to the planes. There are at least two possible minimal surfaces in the slab between the planes and whose boundaries are $C:=C_{1} \cup C_{2}$ : a piece of a catenoid or the surface consisting of the two discs $D_{1}$ and $D_{2}$ determined by the circles (see [Shi56]). This situation is depicted in Figure 1.1. If the planes are too close together, then there are two catenoids whose boundaries are $C$, and the pair of discs has a larger area then the catenoids. Thus, $D_{1} \cup D_{2}$ cannot be area minimising, despite being a minimal surface. 


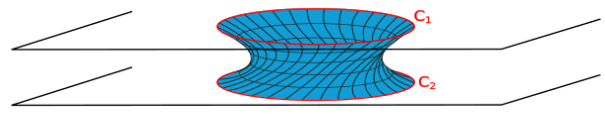

(a) A minimal annulus with boundary given by $C$.

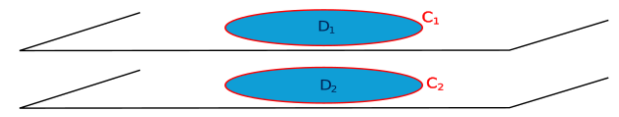

(b) The flat discs whose boundaries are $C_{1}$ and $C_{2}$.

Figure 1.1: Two minimal surfaces with the same boundary.

The right description of minimal surfaces are as critical points for the area functional under suitable constraints. In the examples above, the surfaces need to have a prescribed boundary. If we only consider closed surfaces, no constraints are necessary. However, there is a third situation that can be studied: when we consider compact surfaces whose boundaries are allowed to move freely in the boundary of the ambient manifold. Not surprisingly, critical points of the area functional under this constraint are called free boundary minimal surfaces. The boundary of such surfaces meet the boundary of the ambient manifold at a $90^{\circ}$ angle.

Although the first works dealing with this type of surfaces date back to 1938 with R. Courant (see [Cou38] and [Cou50]), in the last decade there have been incredible developments in this field, with the employment of new techniques and the emergence of interesting conceptual links. Among the main contributors to this topic of research we could cite Fraser, Chen and Pang, with their work on free boundary surfaces on positively curved ambients [FP14], Ambrozio with his work on rigidity of mean-convex manifolds [Amb15], and additionally Carlotto and Sharp (jointly with Ambrozio), with their works on compactness analysis and index estimates for free boundary minimal hypersurfaces ([CS18a] and [CS18b]).

This thesis is divided into two parts. We will detail each one of them in the sequence.

In the first part, covered in Chapter 3, we study a natural generalisation of free boundary minimal surfaces, called capillary minimal surfaces. These are critical points of a certain energy functional, which will be presented in Section 3.1. As will be deduced later, they can be characterised as minimal surfaces whose boundary meet the ambient boundary at a constant angle - the capillary angle.

When dealing with the free boundary case, Ambrozio introduced the following functional in the space of compact and properly immersed surfaces in a Riemannian 3-manifold $M$ :

$$
\mathrm{I}(\Sigma)=\frac{1}{2} \inf _{M} R_{M}|\Sigma|+\inf _{\partial M} H_{\partial M}|\partial \Sigma|,
$$

where $R_{M}$ is the scalar curvature of $M, H_{\partial M}$ is the mean curvature of $\partial M,|\Sigma|$ denotes the area of $\Sigma$ and $|\partial \Sigma|$ denotes the length of $\partial \Sigma$.

As a first result, we modify Ambrozio's functional to take care of the capillary case and we show the following theorem, generalising Proposition 6 in [Amb15]:

Theorem A. Let $\left(M^{3}, g\right)$ be a Riemannian 3-manifold with nonempty boundary, and assume that $R_{M}$ and $H_{\partial M}$ are bounded from below. If $\Sigma$ is a compact two sided capillary stable minimal surface, immersed in $M$ with contact angle $\theta \in(0, \pi)$, then

$$
\mathrm{I}_{\theta}(\Sigma):=\frac{1}{2} \inf _{M} R_{M}|\Sigma|+\frac{1}{\sin \theta} \inf _{\partial M} H_{\partial M}|\partial \Sigma| \leq 2 \pi \chi(\Sigma),
$$

where $\chi(\Sigma)$ denotes the Euler characteristic of $\Sigma$. Moreover, equality occurs if and only if $\Sigma$ satisfies the following properties:

(i) $\Sigma$ is totally geodesic in $M$ and the geodesic curvature of $\partial \Sigma$ in $\partial M$ is equal to $(\cot \theta)$ inf $H_{\partial M}$;

(ii) the scalar curvature $R_{M}$ is constant along $\Sigma$ and equal to inf $R_{M}$, and the mean curvature $H_{\partial M}$ is constant along $\partial \Sigma$ and equal to inf $H_{\partial M}$; 
(iii) $\operatorname{Ric}(N)=0$ and $\mathbb{I}(\bar{\nu}, \bar{\nu})=0$, where $N$ is a unit normal for $\Sigma$ and $\mathbb{I}(\bar{\nu}, \bar{\nu})$ denotes the second fundamental form of $\partial M$ in the direction of a unit conormal for $\partial \Sigma$ in $\partial M$.

In particular, (i), (ii) and (iii) imply that $\Sigma$ has constant Gaussian curvature equal to inf $R_{M} / 2$ and $\partial \Sigma$ has constant geodesic curvature equal to inf $H_{\partial M} / \sin \theta$.

A compact two-sided capillary minimal surface, properly embedded in $(M, g)$ with contact angle $\theta \in(0, \pi)$, that satisfies conditions (i), (ii) and (iii) of Theorem A will be called infinitesimally rigid. Given one such surface $\Sigma$, there is a way to obtain a vector field $Z$ in $\Sigma$ such that $g(Z, N)=1$ and $Z$ is tangent to $\partial M$ along $\partial \Sigma$. Let us also denote by $Z$ an extension to $M$ which is tangent to $\partial M$ along the entire boundary of $M$. Let $\phi=\phi(x, t)$ the local flow of $Z$ and fix a number $\alpha$ between 0 and 1 . We show the existence of a local foliation around $\Sigma$, employing the same techniques as Ambrozio in Proposition 10 in [Amb15]:

Theorem B. Let $\left(M^{3}, g\right)$ be a Riemannian 3-manifold with nonempty boundary, and assume that $R_{M}$ and $H_{\partial M}$ are bounded from below. Let $\Sigma$ be a compact two-sided capillary minimal surface, properly embedded in $M$ with contact angle $\theta \in(0, \pi)$. If $\Sigma$ is infinitesimally rigid, then there exists $\varepsilon>0$ and a map $w \in C^{2, \alpha}(\Sigma \times(-\varepsilon, \varepsilon))$ such that for every $t \in(-\varepsilon, \varepsilon)$, the set

$$
\Sigma_{t}=\{\phi(x, w(x, t)): x \in \Sigma\}
$$

is a capillary $C M C$ surface with contact angle $\theta$ and mean curvature $H(t)$. Moreover, for each $x \in \Sigma$ and $t \in(-\varepsilon, \varepsilon)$,

$$
w(x, 0)=0, \quad \int_{\Sigma}(w(\cdot, t)-t) \mathrm{d} A=0, \quad \text { and } \quad \frac{\partial w}{\partial t}(x, 0)=1 .
$$

In particular, taking a smaller $\varepsilon>0$ if necessary, $\left\{\Sigma_{t}\right\}_{t \in(-\varepsilon, \varepsilon)}$ is a capillary CMC foliation of a neighbourhood of $\Sigma$ in $M$.

It is also possible to show the existence of a capillary minimal foliation, where the contact angles now vary from leaf to leaf. Although it will not be used subsequently, we believe it may be of independent interest.

Theorem C. Let $\left(M^{3}, g\right)$ be a Riemannian 3-manifold with nonempty boundary, and assume that $R_{M}$ and $H_{\partial M}$ are bounded from below. Let $\Sigma$ be a compact two-sided capillary minimal surface, properly embedded in $M$ with contact angle $\theta \in(0, \pi)$. If $\Sigma$ is infinitesimally rigid, then there exists $\varepsilon>0$ and a map $w \in C^{2, \alpha}(\Sigma \times(-\varepsilon, \varepsilon))$ such that for every $t \in(-\varepsilon, \varepsilon)$, the set

$$
\Sigma_{t}=\{\phi(x, w(x, t)): x \in \Sigma\}
$$

is a capillary minimal surface with contact angle $\theta(t) \in(0, \pi)$. Moreover, for each $x \in \Sigma$ and $t \in(-\varepsilon, \varepsilon)$,

$$
w(x, 0)=0, \quad \int_{\Sigma}(w(\cdot, t)-t) \mathrm{d} A=0, \quad \text { and } \quad \frac{\partial w}{\partial t}(x, 0)=1 .
$$

In particular, taking a smaller $\varepsilon>0$ if necessary, $\left\{\Sigma_{t}\right\}_{t \in(-\varepsilon, \varepsilon)}$ is a capillary minimal foliation of a neighbourhood of $\Sigma$ in $M$.

Then, we use Theorem B to show that, under some hypotheses, a dichotomy occurs: either the contact angle is equal to $\pi / 2$ or a very special situation takes place. More precisely, we have:

Theorem D. Let $\left(M^{3}, g\right)$ be a Riemannian 3-manifold with nonempty and weakly mean-convex boundary, and assume that $R_{M}$ is bounded from below. Let $\Sigma$ be an energy-minimising and infinitesimally rigid surface, properly embedded in $M$ with contact angle $\theta \in(0, \pi)$. Assume that one of the following hypothesis holds: 
(a) each component of $\partial \Sigma$ is locally length-minimising in $\partial M$; or

(b) $\inf _{\partial M} H_{\partial M}=0$.

Then either $\theta=\pi / 2$ or $\Sigma$ is a flat and totally geodesic cylinder, $M$ is flat and $\partial M$ is totally geodesic around $\Sigma$. In the first case, there is a neighbourhood of $\Sigma$ in $M$ that is isometric to $(\Sigma \times$ $\left.(-\varepsilon, \varepsilon), g_{\Sigma}+\mathrm{d} t^{2}\right)$, where $\left(\Sigma, g_{\Sigma}\right)$ has constant Gaussian curvature inf $R_{M} / 2$ and $\partial \Sigma$ has constant geodesic curvature inf $H_{\partial M}$ in $\Sigma$.

A situation when $\theta \neq \pi / 2$ may happen in Theorem $\mathrm{D}$ is the following. Let $P_{1}$ and $P_{2}$ be two non parallel planes in $\mathbb{R}^{3}$, intersecting along a line $\ell$, and let $Q$ be a plane in $\mathbb{R}^{3}$ which is parallel to $\ell$ and intersects both $P_{1}$ and $P_{2}$ at the same angle. Fix $S_{0}$ to be the (closed) wedge determined by $P_{1}$ and $P_{2}$ which contains both $Q \cap P_{1}$ and $Q \cap P_{2}$, and let $S=S_{0} \backslash \ell$ (see Figure 1.2). Now fix $T$ a translation of $\mathbb{R}^{3}$ by a vector parallel to the line $\ell$ and let $M$ be the quotient of $S$ by the group $G$ generated by $T$. If we define $\Sigma$ to be the quotient of $Q \cap S$ by $G$, then $\Sigma$ is an infinitesimally rigid cylinder intersecting $\partial M$ at a constant angle, (a) and (b) hold, $M$ is flat and $\partial M$ is totally geodesic, as we wanted. One question remains: is $\Sigma$ energy-minimising? We believe so, but we did not find a proof.

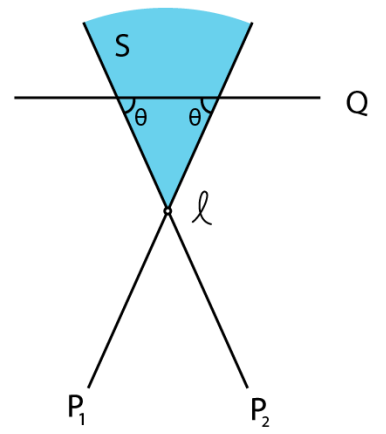

Figure 1.2: An arrangement of 3 planes in $\mathbb{R}^{3}$ seen from a plane orthogonal to the line $\ell$.

Next, we prove two other inequalities relating the geometry and the topology of capillary minimal surfaces of low index. This result generalises Theorem 1.2 in [FP14] to the capillary case. We note that item (i) below also generalises the free boundary case.

Theorem E. Let $\left(M^{3}, g\right)$ be a Riemannian 3-manifold with nonempty boundary. Suppose that $\Sigma$ is a compact orientable two-sided capillary minimal surface of genus $g$ and with $k \geq 1$ boundary components, immersed in $M$ with contact angle $\theta \in(0, \pi)$.

(i) Suppose that $(M, g)$ has nonnegative Ricci curvature and weakly mean-convex boundary. If $\Sigma$ has index 1 then

$$
\int_{\partial \Sigma} k_{g} \mathrm{~d} L<2 \pi\left[9-(-1)^{g}-2(g+k)\right] .
$$

In particular, if the total geodesic curvature of $\partial \Sigma$ (in $\Sigma$ ) is nonnegative (which happens if $\Sigma$ is free boundary and $\partial M$ is weakly convex, for instance), then

(a) $g+k \leq 3$ if $g$ is even;

(b) $g+k \leq 4$ if $g$ is odd.

(ii) Suppose that the scalar curvature of $(M, g)$ and the mean curvature of $\partial M$ are bounded from below. If $\Sigma$ has index 1 , then

$$
\mathrm{I}_{\theta}(\Sigma)=\frac{1}{2} \inf _{M} R_{M}|\Sigma|+\frac{1}{\sin \theta} \inf _{\partial M} H_{\partial M}|\partial \Sigma|<2 \pi\left[7-(-1)^{g}-k\right] .
$$


(iii) Suppose that $(M, g)$ has scalar curvature $R_{M} \geq R_{0}>0$ and weakly mean-convex boundary.

(a) If $\Sigma$ is stable, then it is a disc and $|\Sigma| \leq \frac{4 \pi}{R_{0}}$.

(b) If $\Sigma$ has index 1 , then $|\Sigma| \leq \frac{4 \pi\left[7-(-1)^{g}-k\right]}{R_{0}}$.

In the second part of this thesis, contained in Chapter 4, we will deal with homological systoles in 3-manifolds with boundary. The results therein were published by the author in the paper [Lon20].

Systolic Geometry dates back to the late 1940s, with the work of Charles Loewner and his doctoral student Pao Ming $\mathrm{Pu}$. This branch of differential geometry received more attention after the seminal work of Gromov [Gro83], where he proved his famous systolic inequality and introduced many concepts, notably the filling radius and the filling volume of a manifold. This line of research would be popularised, subsequently, by Marcel Berger, in a series of books and articles (see [Ber], [Ber03], [Ber08], for example).

The main objects of Systolic Geometry are, as one would expect, the systoles. The first work in the field ([Pu52] and a result by C. Loewner cited there, unpublished) dealt with the so called homotopic systole. In words, this is the infimum of all lengths of closed curves that cannot be continuously shrunk to a point (see Figure 1.3). Notice that if this systole is attained by the length of a closed curve, then such a curve must be a geodesic. It should be somewhat clear from the picture that if the systole of the torus is large, then its area cannot be too small. It is thus natural to explore the relation between these quantities. Many results in this direction have been obtained, and we refer the reader to the book of Berger [Ber03] to check a list of them.

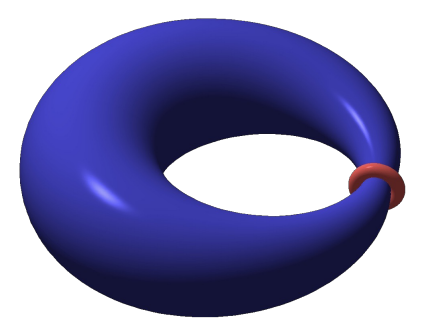

Figure 1.3: The systole is realised be the red curve in this torus.

As any mathematician, we could ask for a generalisation of the concept of homotopic systole. One possible direction to go - and this is what we are interested in - is to to pass from curves to surfaces and submanifolds. Geometers have then come up with the notion of homological systole. Roughly speaking, the homological $k$-systole of a Riemannian $n$-manifold $M$, denoted by $\operatorname{sys}_{k}(M)$, is the least ( $k$-dimensional) volume of a nontrivial closed submanifold of dimension $k$. By nontrivial here we mean nontrivial in homology, which is the right notion of noncontractible from the algebraic topology point of view. As is the case of curves, notice that if the homological systole is realised by the volume of a closed submanifold, then the latter must be minimal, since homology classes are open in the space of embeddings of a fixed submanifold.

In this work, we will deal with homological systoles in Riemannian 3-manifolds with nonempty boundary. Let $\left(M^{3}, g\right)$ be such a manifold. Define its relative homological 2-systole by

$$
\operatorname{sys}_{2}(M, \partial M)=\inf \left\{\operatorname{Area}(\Sigma): \Sigma \in \mathcal{S} \text { and }[\Sigma] \neq 0 \in H_{2}(M, \partial M ; \mathbb{Z})\right\}
$$

where $\mathcal{S}$ denotes the set of all compact and embedded surfaces $\Sigma \subset M$ with boundary such that $\Sigma \cap \partial M=\partial \Sigma$. Notice that any surface in $\mathcal{S}$ that represents a nonzero element of $H_{2}(M, \partial M ; \mathbb{Z})$ must be orientable. We also define the boundary homology 1-systole of $\partial M$ by

$$
\operatorname{sys}_{1}^{\partial}(\partial M)=\inf \left\{\operatorname{Length}(\partial \Sigma): \Sigma \in \mathcal{S} \text { and }[\partial \Sigma] \neq 0 \in H_{1}(\partial M ; \mathbb{Z})\right\} .
$$

In the sequel, we present sharp inequalities relating the (relative) homological systoles of a Riemannian 3-manifold $\left(M^{3}, g\right)$ to its scalar curvature and mean curvature of the boundary. In all 
the results that follow, we assume that the scalar curvature of $M$ is positive (or nonnegative) and that $\partial M$ is mean-convex (or weakly mean-convex). Having this in mind, it is important to further elucidate the setting in which our results apply.

Recently, Carlotto and Li proved a complete topological classification of those compact, connected and orientable 3-manifolds with boundary which support Riemannian metrics of positive scalar curvature and mean-convex boundary (see Theorem 1.1 and Corollary 2.6 in [CL]). Namely, if $M^{3}$ is such a manifold, then there exist integers $A, B, C, D \geq 0$ such that $M$ is diffeomorphic to a connected sum of the form

$$
P_{\gamma_{1}} \# \cdots \# P_{\gamma_{A}} \# \mathbb{S}^{3} / \Gamma_{1} \# \cdots \# \mathbb{S}^{3} / \Gamma_{B} \#\left(\# \#_{i=1}^{C} \mathbb{S}^{2} \times \mathbb{S}^{1}\right) \backslash\left(\sqcup_{i=1}^{D} B_{i}^{3}\right),
$$

where $P_{\gamma_{i}}, i \leq A$, are genus $\gamma_{i}$ handlebodies; $\Gamma_{i}, i \leq B$, are finite subgroups of $S O(4)$ acting freely on $\mathbb{S}^{3} ; B_{i}^{3}, i \leq D$, are disjoint 3-balls in the interior. Conversely, if $M$ is of this form, then it supports Riemannian metrics of positive scalar curvature and mean-convex boundary.

The first theorem of this second part is the counterpart of Theorem 1.6 in [Ste] (originally proved by Bray-Brendle-Neves in a stronger version [HBN10b]) for manifolds with boundary. It exhibits an explicit relation between the relative homological 2-systole of a Riemannian 3-manifold and its scalar curvature.

Theorem F. Let $\left(M^{3}, g\right)$ be a compact, connected and oriented Riemannian 3-manifold with nonempty boundary. Assume that $H_{2}(M, \partial M ; \mathbb{Z}) \neq 0$. If $M$ has positive scalar curvature $\left(R_{M}>0\right)$ and weakly mean-convex boundary $\left(H_{\partial M} \geq 0\right)$, then

$$
\operatorname{sys}_{2}(M, \partial M) \inf _{M} R_{M} \leq 4 \pi .
$$

Moreover, if equality holds, then the universal cover of $M$ is isometric to the cylinder $\mathbb{S}_{+}^{2} \times \mathbb{R}$ up to scaling, where $\mathbb{S}_{+}^{2}$ is a closed hemisphere of the unit round sphere.

A natural question in whether the number $\operatorname{sys}_{2}(M, \partial M)$ is attained by the area of a compact and properly embedded surface $\Sigma \subset M$. To answer this question, we first introduce some terminology. Recall that an embedded surface $\Sigma \subset M$ is said to be separating if $M \backslash \Sigma$ has at least two connected components. We say that a compact and orientable 3-manifold $M$ is weakly irreducible if every smoothly embedded 2-sphere in the interior of $M$ is separating.

We then answer affirmatively the above question assuming some hypothesis on the geometry and topology of $M$ :

Theorem G. Let $\left(M^{3}, g\right)$ be a compact, connected and oriented Riemannian 3-manifold with nonempty boundary. Assume that $M$ is weakly irreducible and $H_{2}(M, \partial M ; \mathbb{Z}) \neq 0$. If $M$ has positive scalar curvature and weakly mean-convex boundary, then there exists a properly embedded free boundary stable minimal disc $D$ in $M$ such that $[D] \neq 0$ in $H_{2}(M, \partial M ; \mathbb{Z})$ and $\operatorname{Area}(D)=\operatorname{sys}_{2}(M, \partial M)$.

See the comments made after the proof of this theorem in Section 4.3 for a careful analysis of what 3-manifolds satisfy the hypothesis of Theorem G.

Using the same techniques as in Theorem F, we also prove:

Theorem H. Let $\left(M^{3}, g\right)$ be a compact, connected and oriented Riemannian 3-manifold with nonempty boundary. Assume that $M$ is weakly irreducible and that $H_{2}(M, \partial M ; \mathbb{Z}) \neq 0$. If $M$ has positive scalar curvature and weakly mean-convex boundary, then

$$
\frac{1}{2} \operatorname{sys}_{2}(M, \partial M) \inf _{M} R_{M}+\operatorname{sys}_{1}^{\partial}(\partial M) \inf _{\partial M} H_{\partial M} \leq 2 \pi .
$$

Moreover, if equality holds, then the universal cover of $M$ is isometric to the cylinder $\mathbb{B}_{r}^{2} \times \mathbb{R}$ up to scaling, where $\mathbb{B}_{r}^{2}$ is a geodesic ball of radius $r=\cos ^{-1}\left(1-\frac{\operatorname{sys}_{2}(M, \partial M)}{2 \pi}\right)$ of the unit round sphere. 
It is worth mentioning that, just as Theorem 1.6 in [Ste] is similar to Theorem 1 in [HBN10b], Theorem $\mathrm{H}$ resembles the rigidity results of [Amb15]. Inarguably, the geometric invariants in [Amb15, Theorem 8] and in Theorem $\mathrm{H}$ have similar expressions. Nonetheless, whereas we minimise area over all compact surfaces with boundary which are nontrivial in (relative) homology in order to define $\operatorname{sys}_{2}(M, \partial M)$, Ambrozio minimises area over the set of all discs whose boundaries are curves in $\partial M$ that are homotopically nontrivial in $\partial M$ in order to define $\mathcal{A}(M, g)$. This may produce different results, as Figure 1.4 shows.

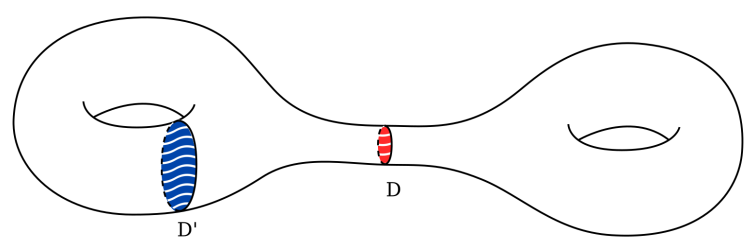

Figure 1.4: The disc $D$ realises the quantity $\mathcal{A}(M, g)$, whereas $D^{\prime}$ realises $\operatorname{sys}_{2}(M, \partial M)$.

Finally, making a slight adaptation on the proof of Theorem H, we obtain the following corollary:

Corollary I. Let $\left(M^{3}, g\right)$ be a compact, connected and oriented Riemannian 3-manifold with nonempty boundary. Assume that $M$ is weakly irreducible and that $H_{2}(M, \partial M ; \mathbb{Z}) \neq 0$. If $M$ has nonnegative scalar curvature and mean-convex boundary then

$$
\operatorname{sys}_{1}^{\partial}(\partial M) \inf _{\partial M} H_{\partial M} \leq 2 \pi \text {. }
$$

Moreover, if equality holds, then the universal cover of $M$ is isometric to the right circular cylinder $\mathbb{D}^{2} \times \mathbb{R}$ up to scaling, where $\mathbb{D}^{2}$ is the flat closed unit disc. 


\section{Chapter 2}

\section{Geometric preliminaries}

The objectives of this short chapter are both fixing notation and establishing conventions. From now on, all manifolds and maps between them are assumed to be smooth.

\subsection{Riemannian Geometry}

Let $\left(M^{n}, g\right)$ be a Riemannian manifold with Levi-Civita connection $\nabla$. The curvature tensor of $M$ is given by

$$
R(X, Y) Z=\nabla_{Y} \nabla_{X} Z-\nabla_{X} \nabla_{Y} Z+\nabla_{[X, Y]} Z
$$

for any three vector fields $X, Y, Z$ on $M$.

Fix a point $p \in M$. Given two linearly independent tangent vectors $X, Y \in T_{p} M$, the sectional curvature of $M$ at the 2-plane determined by $X$ and $Y$ is given by

$$
K(X \wedge Y)=\frac{g(R(X, Y) X, Y)}{g(X, X) g(Y, Y)-g(X, Y)} .
$$

For any two tangent vectors $X, Y \in T_{p} M$, the Ricci curvature of $M$ at $p$ calculated on $(X, Y)$ is

$$
\operatorname{Ric}(X, Y)=\sum_{i=1}^{n} g\left(R\left(X, E_{i}\right) Y, E_{i}\right),
$$

where $\left\{E_{1}, \ldots, E_{n}\right\}$ is an orthonormal basis of $T_{p} M$. We write $\operatorname{Ric}(X)$ to denote $\operatorname{Ric}(X, X)$.

The scalar curvature of $M$ is the function $R_{M}: M \rightarrow \mathbb{R}$ given by

$$
R_{M}(p)=\sum_{i=1}^{n} \operatorname{Ric}\left(E_{i}\right)
$$

where $\left\{E_{1}, \ldots, E_{n}\right\}$ is as above.

Now let $\varphi: \Sigma^{n-1} \rightarrow M$ be an immersion of a smooth $(n-1)$-manifold $\Sigma$. We say that $\varphi$ (or $\Sigma$, by an abuse of language) is two-sided if its normal bundle in $M$ is trivial. Since this is a line bundle over $\Sigma$, triviality is equivalent to the existence of a global section, i.e. a normal vector field along $\varphi$. This is always the case when $\Sigma$ is simply connected, for instance.

The following notions are local, so we identify small pieces of $\Sigma$ and with their images via $\varphi$, and tangent spaces of $\Sigma$ with their images via d $\varphi$. Let $N$ be a normal vector field for $\Sigma$ defined in a neighbourhood $U \subseteq \Sigma: N(p) \perp T_{p} \Sigma$, for all $p \in U$. The second fundamental form of $\Sigma$ at $p \in U$ is the symmetric bilinear form $A_{p}: T_{p} \Sigma \rightarrow T_{p} \Sigma \rightarrow \mathbb{R}$ defined by

$$
A_{p}(X, Y)=g\left(\nabla_{\bar{X}} \bar{Y}, N\right), \quad X, Y \in T_{p} \Sigma,
$$


where $\bar{X}$ and $\bar{Y}$ are local extensions of $X$ and $Y$ to $M$. The squared norm of the second fundamental form is the quantity

$$
\left\|A_{p}\right\|^{2}=\sum_{i=1}^{n-1} A_{p}\left(E_{i}, E_{j}\right)^{2}, \quad p \in U,
$$

where $\left\{E_{1}, \ldots, E_{n-1}\right\}$ is an orthonormal basis of $T_{p} \Sigma$. If $A \equiv 0, \Sigma$ is called totally geodesic.

The shape operator of $\Sigma$ at $p \in U$ with respect to the normal direction $N$ is the endomorphism $S_{p}: T_{p} \Sigma \rightarrow T_{p} \Sigma$ given by

$$
S_{p}(X)=-\left(\nabla_{X} N\right)^{\top}, \quad X \in T_{p} M,
$$

where $(\cdot)^{\top}$ denotes the orthogonal projection onto $T_{p} \Sigma$. Notice that $S_{p}$ is the self-adjoint operator associated with the symmetric bilinear form $A_{p}$, that is,

$$
g\left(S_{p}(X), Y\right)=A_{p}(X, Y)
$$

for any tangent vectors $X, Y \in T_{p} \Sigma$.

The mean curvature of $\Sigma$ is the function $H: \Sigma \rightarrow \mathbb{R}$ given by

$$
H(p)=\operatorname{trace} S_{p}=\sum_{i=1}^{n-1} \lambda_{i}(p), \quad p \in \Sigma,
$$

where $\left\{\lambda_{1}(p), \ldots, \lambda_{n-1}(p)\right\}$ are the eigenvalues of $S_{p}$, which are called the principal curvatures of $\Sigma$. In terms of principal curvatures, the squared norm of the second fundamental form $\Sigma$ is given by

$$
\left\|A_{p}\right\|^{2}=\sum_{i=1}^{n-1} \lambda_{i}^{2}(p), \quad p \in U .
$$

Finally, let us recall the Gauss-Bonnet theorem for compact surfaces. First, some terminology. If $(\Sigma, g)$ is a Riemannian surface, let $K_{\Sigma}$ denote its Gaussian curvature; if the boundary of $\Sigma$ is nonempty, let $k_{g}$ be its geodesic curvature, defined as $k_{g}=g\left(\nabla_{T} \nu, T\right)$, where $\nu$ is the unit outward conormal to $\partial \Sigma$ and $T$ is a unit tangent vector field along $\partial \Sigma$.

Theorem (Gauss-Bonnet). Let $\Sigma$ be a compact Riemannian surface. Then

$$
\int_{\Sigma} K_{\Sigma} \mathrm{d} A+\int_{\partial \Sigma} k_{g} \mathrm{~d} L=2 \pi \chi(\Sigma)
$$

where $\mathrm{d} A$ is the area element of $\Sigma, \mathrm{d} L$ is the line element of $\partial \Sigma$ and $\chi(\Sigma)$ denotes the Euler characteristic of $\Sigma$.

We remark that if $\Sigma$ is compact orientable of genus $g \geq 0$ and with $k \geq 1$ boundary components, then $\chi(\Sigma)=2-2 g-k$.

\section{$2.2 \quad$ Algebraic Topology}

Let $(X, A)$ be a pair of topological spaces. The $n$-th singular homology group (resp. cohomology group) of $(X, A)$ with coefficients in an abelian group $G$ will be denoted by $H_{n}(X, A ; G)$ (resp. $\left.H^{n}(X, A ; G)\right)$. When $A=\emptyset$ we just write $H_{n}(X)$ or $H^{n}(X)$. By convention, $H_{n}(X, A ; G)=0$ whenever $n<0$. Given a map $f:(X, A) \rightarrow(Y, B)$ between pairs of spaces, the induced homomorphism in homology (resp. cohomology) is indicated by $f_{*}: H_{n}(X, A ; G) \rightarrow H_{n}(Y, B ; G)$ (resp. $\left.f^{*}: H^{n}(Y, B ; G) \rightarrow H^{n}(X, A ; G)\right)$. 
For any pair $(X, A)$ there is a long exact sequence in homology of the form

$$
\cdots \longrightarrow H_{n}(A) \stackrel{i_{*}}{\longrightarrow} H_{n}(X) \stackrel{j_{*}}{\longrightarrow} H_{n}(X, A) \stackrel{\partial}{\longrightarrow} H_{n-1}(A) \longrightarrow \cdots
$$

where $i:(A, \emptyset) \rightarrow(X, \emptyset)$ and $j:(X, \emptyset) \rightarrow(X, A)$ are the inclusion maps and $\partial: H_{n}(X, A) \rightarrow$ $H_{n-1}(A)$ is called the connecting homomorphism in homology. The long exact sequence in cohomology is similar, but the arrows go in the other direction.

Let us recall an important theorem for relating cohomology to homology.

Theorem (Universal Coefficient Theorem). Let $(X, A)$ be a pair of topological spaces and let $G$ be an abelian group. Then there is a short exact sequence

$$
0 \longrightarrow \operatorname{Ext}\left(H_{n-1}(X, A ; \mathbb{Z}), G\right) \longrightarrow H^{n}(X, A ; G) \longrightarrow \operatorname{Hom}\left(H_{n}(X, A ; \mathbb{Z}), G\right) \longrightarrow 0
$$

for each $n \geq 0$. Furthermore, the sequences split, but not naturally.

Finally, let us state the Künneth Theorem for computing the homology groups of a product.

Theorem (Künneth Theorem). Let $X$ and $Y$ be topological spaces and let $G$ be an abelian group. Then there is a short exact sequence

$$
0 \longrightarrow \bigoplus_{i+j=n} H_{i}(X ; G) \otimes H_{j}(Y ; G) \rightarrow H_{n}(X \times Y ; G) \rightarrow \bigoplus_{i+j=n-1} \operatorname{Tor}\left(H_{i}(X ; G), H_{j}(Y ; G)\right) \longrightarrow 0
$$

for each $n \geq 0$. Furthermore, the sequences split, but not naturally. In particular, if the homology groups of $X$ and $Y$ with coeffients in $G$ are torsion free, then

$$
H_{n}(X \times Y ; G) \cong \bigoplus_{i+j=n} H_{i}(X ; G) \otimes H_{j}(Y ; G), \quad n \geq 0
$$




\section{Chapter 3}

\section{Capillary surfaces}

Let $\Omega$ be a compact domain in $\mathbb{R}^{3}$ having smooth boundary. A capillary CMC (resp. minimal) surface in $\Omega$ is a compact CMC (resp. minimal) surface with nonempty boundary, which is contained in $\Omega$ and meets $\partial \Omega$ at a constant angle along its boundary.

Capillary surfaces in $\mathbb{R}^{3}$ model the configuration of liquids in containers in the absence of gravity. In fact, the interface between the fluid and the air is a surface with boundary that minimises (locally) the energy functional that will be introduced in Section 3.1. The energy depends on the area of the interface, the area wetted by the fluid in the container and the angle of contact between the surface and the boundary of the container. More general situations have also been considered in the literature, like the influence of gravity and the density of the fluid in the equilibrium shape. We refer the interested reader to the book of Finn [Fin86] for an extensive survey on this subject and the derivation of the equations that describe such surfaces.

Like in the free boundary case, questions relating the topology and the geometry of surfaces raise a lot of attention from geometers. For instance, given an ambient manifold $\left(M^{3}, g\right)$ of a particular shape, what are the possible topological types of surfaces $\Sigma$ that admit a capillary CMC or minimal embedding into $M$ ? Is it possible to characterise the geometry of the allowed types? The first result in this direction was obtained by Nitsche [Nit85], who proved that any immersed capillary disc in the unit ball of $\mathbb{R}^{3}$ must be either a spherical cap or a flat disc. Later, Ros and Souam [RS97] extended this result to capillary discs in balls of 3-dimensional space forms. Recently, Wang and Xia [WX19] analysed the problem in an arbitrary dimension and proved that any stable immersed capillary hypersurface in a ball in space forms is totally umbilical. There are many interesting uniqueness results in other types of domains, like slabs [AS16], wedges [Par05, CK16], cylinders [Lí7] and cones [RR04].

We are interested in the same questions raised above, but in a more general setting. Namely, we only impose curvature assumptions on the ambient 3-manifold and look for restrictions in the topology of the possible immersed (or embedded) capillary minimal surfaces. We will recall the results we obtained and prove them in Section 3.2.

\subsection{Preliminaries: variational problem and stability}

The purpose of this section is to formally introduce the concept of capillary CMC and minimal hypersurfaces. Despite the fact that in section 3.2 we will be dealing only with capillary surfaces in Riemannian 3-manifolds, there is no significant simplification in introducing the main concepts only in dimension 2 . So, the general situation will be addresed in the sequel.

Let $\left(M^{n+1}, g\right)$ be a Riemannian manifold with nonempty boundary. Let $\Sigma^{n}$ be a smooth compact manifold with nonempty boundary, and let $\varphi: \Sigma \rightarrow M$ be a smooth immersion of $\Sigma$ into $M$. We say that $\varphi$ is a proper immersion if $\varphi(\Sigma) \cap \partial M=\varphi(\partial \Sigma)$.

Henceforth, we assume that $\varphi$ is two-sided. Fix a unit normal vector field $N$ for $\Sigma$ along $\varphi$ and denote by $\nu$ the outward unit conormal for $\partial \Sigma$ in $\Sigma$. Moreover, let $\bar{N}$ the outward pointing unit 
normal for $\partial M$ and let $\bar{\nu}$ the unit normal for $\partial \Sigma$ in $\partial M$ such that the bases $\{N, \nu\}$ and $\{\bar{N}, \bar{\nu}\}$ determine the same orientation in $(T \partial \Sigma)^{\perp}$. See Figure 3.1 to gain some intuition.

A smooth function $\Phi: \Sigma \times(-\varepsilon, \varepsilon) \rightarrow M$ is called a proper variation of $\varphi$ is the maps $\varphi_{t}: \Sigma \rightarrow M$, defined by $\varphi_{t}(x)=\Phi(x, t)$, are proper immersions for all $t \in(-\varepsilon, \varepsilon)$, and if $\varphi_{0}=\varphi$.

Let us fix a proper variation $\Phi$ of $\varphi$. The variational vector field associated to $\Phi$ is the vector field $\xi_{\Phi}=\xi: \Sigma \rightarrow T M$ along $\varphi$ defined by

$$
\xi(x)=\frac{\partial \Phi}{\partial t}(x, 0), \quad x \in \Sigma .
$$

We now define some important functionals related to the variation $\Phi$. The area functional $A:(-\varepsilon, \varepsilon) \rightarrow \mathbb{R}$ is given by

$$
A(t)=\int_{\Sigma} \mathrm{d} A_{\varphi_{t}^{*} g}
$$

where $\mathrm{d} A_{\varphi_{t}^{*} g}$ denotes the area element of $\left(\Sigma, \varphi_{t}^{*} g\right)$. Even if $n=\operatorname{dim} \Sigma>2$, it is customary to refer to this as the area functional.

The volume functional $V:(-\varepsilon, \varepsilon) \rightarrow \mathbb{R}$ is defined by

$$
V(t)=\int_{\Sigma \times[0, t]} \Phi^{*}(\mathrm{~d} V)
$$

where $\mathrm{d} V$ is the volume element of $M$. We say that the variation $\Phi$ is volume preserving if $V(t)=0$ for every $t \in(-\varepsilon, \varepsilon)$.

We also consider the wetting area functional $W:(-\varepsilon, \varepsilon) \rightarrow \mathbb{R}$ :

$$
W(t)=\int_{\partial \Sigma \times[0, t]} \Phi^{*}\left(\mathrm{~d} A_{\partial M}\right)
$$

where $\mathrm{d} A_{\partial M}$ denotes the area element of $\partial M$.

Finally, we define the energy functional. In order to do so, let us fix an angle $\theta \in(0, \pi)$. Then $E_{\Phi, \theta}=E:(-\varepsilon, \varepsilon) \rightarrow \mathbb{R}$ is given by

$$
E(t)=A(t)-(\cos \theta) W(t) .
$$

The following proposition contains the formulae for the first variation of the energy and volume, whose proof can be found in [Spi99] and [JLBE88]:

Proposition 3.1.1. Let $\Phi$ be a proper variation of the immersion $\varphi: \Sigma \rightarrow M$. Then the following formulae hold:

$$
\begin{aligned}
E^{\prime}(0) & =-\int_{\Sigma} H f \mathrm{~d} A+\int_{\partial \Sigma} g(\xi, \nu-(\cos \theta) \bar{\nu}) \mathrm{d} L \quad \text { and } \\
V^{\prime}(0) & =\int_{\Sigma} f \mathrm{~d} A
\end{aligned}
$$

where $f=g(\xi, N), H$ is the mean curvature of $\Sigma$ with respect to $N, \mathrm{~d} A$ is the area element of $\Sigma$ induced by $\varphi$ and $\mathrm{d} L$ is the line element of $\partial \Sigma$ induced by $\varphi$.

We say that the immersion $\varphi$ is a capillary $C M C$ immersion if $E^{\prime}(0)=0$ for every volume preserving variation of $\varphi$. If $E^{\prime}(0)=0$ for every variation of $\varphi$, we call $\varphi$ a capillary minimal immersion. When there is only one immersion under consideration and there is no risk of confusion, we just say that $\Sigma$ is a capillary CMC or minimal hypersurface. See Figure 3.1 for an example in an Eucliean cone.

Notice that $\Sigma$ is a capillary CMC hypersurface if and only if $\Sigma$ has constant mean curvature and $g(N, \bar{N})=\cos \theta$ along $\partial \Sigma$; this last condition means that $\partial \Sigma$ meets $\partial M$ at an angle of $\theta$. Similarly, 


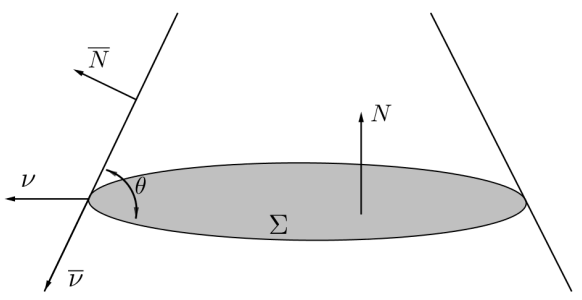

Figure 3.1: The four fundamental vector fields in a capillary minimal surface in a cone.

$\Sigma$ is a capillary minimal hypersurface when $\Sigma$ is a minimal hypersurface and $\partial \Sigma$ meets $\partial M$ at an angle of $\theta$. When $\theta=\pi / 2$, we use the term free boundary CMC (or minimal) hypersurface.

For a capillary CMC or minimal hypersuface $\Sigma$ with contact angle $\theta \in(0, \pi)$, the orthonormal bases $\{N, \nu\}$ and $\{\bar{N}, \bar{\nu}\}$ are related by the following equations:

$$
\left\{\begin{array}{l}
\bar{N}=(\cos \theta) N+(\sin \theta) \nu \\
\bar{\nu}=-(\sin \theta) N+(\cos \theta) \nu
\end{array}\right.
$$

This will be important in the calculations.

Proposition 3.1.2. [AS16, Proposition 2.1] Let $\varphi: \Sigma^{n} \rightarrow M^{n+1}$ be a proper immersion which is transversal to $\partial M$. For any smooth function $f: \Sigma \rightarrow \mathbb{R}$ satisfying $\int_{\Sigma} f \mathrm{~d} A=0$, there exists a volume preserving variation $\Phi$ of $\varphi$ such that $f=g\left(\xi_{\Phi}, N\right)$, where $\xi=\left.\frac{\partial \Phi}{\partial t}\right|_{t=0}$ is the variational vector field associated to $\Phi$. If we don't assume that $\int_{\Sigma} f \mathrm{~d} A=0$, then the result still holds, but now $\Phi$ doesn't need to be volume preserving.

We now discuss the notion of stability. Firstly, let us fix some notation. For a proper immersion $\varphi: \Sigma \rightarrow M$, let $A(X, Y)=g\left(-\nabla_{X} N, Y\right)$ the second fundamental form of $\Sigma$, and denote by $\Delta_{\Sigma}$ the Laplacian of $\Sigma$ with respect to the metric induced by $\varphi$. Moreover, let $\mathbb{I}(v, w)=g\left(\nabla_{v} \bar{N}, w\right)$ be the second fundamental form of $\partial M$ with respect to $-\bar{N}$.

The following proposition gives a formula for the second variation of energy. The proof is long and can be found in the Appendix of [RS97].

Proposition 3.1.3. Let $\varphi: \Sigma \rightarrow M$ be a capillary CMC immersion and let $f: \Sigma \rightarrow \mathbb{R}$ be a smooth function which satisfies $\int_{\Sigma} f \mathrm{~d} A=0$. Let $\Phi$ be a volume preserving proper variation of $\varphi$ such that $f=g\left(\xi_{\Phi}, N\right)$ (see Proposition 3.1.2). Then,

$$
\begin{aligned}
E^{\prime \prime}(0) & =-\int_{\Sigma}\left[\Delta_{\Sigma} f+\left(\operatorname{Ric}(N)+\|A\|^{2}\right) f\right] f \mathrm{~d} A+\int_{\partial \Sigma}\left(\frac{\partial f}{\partial \nu}-q f\right) f \mathrm{~d} L \\
& =-\int_{\Sigma} f L_{\Sigma}(f) \mathrm{d} A+\int_{\partial \Sigma}\left(\frac{\partial f}{\partial \nu}-q f\right) f \mathrm{~d} L
\end{aligned}
$$

where

$$
q=\frac{1}{\sin \theta} \Pi(\bar{\nu}, \bar{\nu})+(\cot \theta) A(\nu, \nu)
$$

and $L_{\Sigma}=\Delta_{\Sigma}+\left(\operatorname{Ric}(N)+\|A\|^{2}\right)$ is the Jacobi operator of $\Sigma$. If $\varphi$ is a capillary minimal immersion and $f$ is any smooth function defined on $\Sigma$, then the same formula holds, but now $\Phi$ doesn't need to be volume preserving.

The capillary CMC immersion $\varphi: \Sigma \rightarrow M$ (or just $\Sigma$ ) is called stable if $E^{\prime \prime}(0) \geq 0$ for any volume preserving variation of $\varphi$. If $\varphi$ is a capillary minimal immersion, we call it stable whenever $E^{\prime \prime}(0) \geq 0$ for every variation of $\varphi$.

Alternatively, let $\mathcal{F}=\left\{f \in H^{1}(\Sigma): \int_{\Sigma} f \mathrm{~d} A=0\right\}$, where $H^{1}(\Sigma)$ is the first Sobolev space of $\Sigma$. 
The index form $Q: H^{1}(\Sigma) \times H^{1}(\Sigma) \rightarrow \mathbb{R}$ of $\Sigma$ is given by

$$
Q(f, h)=\int_{\Sigma}\left[g(\nabla f, \nabla h)-\left(\operatorname{Ric}(N)+\|A\|^{2}\right) f h\right] \mathrm{d} A-\int_{\partial \Sigma} q f h \mathrm{~d} L .
$$

Then $\varphi$ is a capillary CMC stable immersion if and only if $Q(f, f) \geq 0$ for every $f \in \mathcal{F}$. If $\varphi$ is a capillary minimal immersion, then it is stable precisely when $Q(f, f) \geq 0$ for every $f \in H^{1}(\Sigma)$.

The stability index of a capillary CMC (resp. minimal) hypersurface $\Sigma$ is the dimension of the largest vector subspace of $\mathcal{F}$ (resp. $H^{1}(\Sigma)$ ) restricted to which the bilinear form $Q$ is negative definite. Thus, stable hyperfurfaces are those which have index equal to zero. Intuitively, this means that no variation of a capillary minimal stable hypersurface decreases area up to second order.

It is possible to analitically compute the stability index of a capillary CMC (resp. minimal) hypersurface $\Sigma$ in terms of the spectrum of its Jacobi operator with a Robin boundary condition. Indeed, the boundary condition

$$
\frac{\partial f}{\partial \nu}=q f
$$

is elliptic for the Jacobi operator $L_{\Sigma}$. Therefore, there exists a nondecreasing and divergent sequence $\lambda_{1} \leq \lambda_{2} \leq \cdots \leq \lambda_{k} \nearrow \infty$ of eigenvalues for the problem

$$
\begin{cases}L_{\Sigma}(f)+\lambda f=0 & \text { on } \Sigma \\ \frac{\partial f}{\partial \nu}=q f & \text { on } \partial \Sigma\end{cases}
$$

The index of $\Sigma$ is then the number of negative eigenvalues of the problem above associated to (smooth) eigenfunctions that lie in $\mathcal{F}$ (resp. $H^{1}(\Sigma)$ ) (see more details in [FP14] and [Sch06]).

\subsection{Results}

This section is devoted to prove Theorems A, B, D and E mentioned in the Introduction.

Recall that, given a Riemannian 3-manifold $\left(M^{3}, g\right)$ with nonempty boundary and a fixed angle $\theta \in(0, \pi)$, we can define the following functional in the space of compact properly immeresd surfaces in $M$ :

$$
\mathrm{I}_{\theta}(\Sigma)=\frac{1}{2} \inf _{M} R_{M}|\Sigma|+\frac{1}{\sin \theta} \inf _{\partial M} H_{\partial M}|\partial \Sigma|,
$$

provided that the scalar curvature $R_{M}$ and the mean curvature $H_{\partial M}$ are bounded from below.

We first show that if $\Sigma$ is capillary minimal stable, then $\mathrm{I}_{\theta}(\Sigma)$ can be bounded above by the topology of $\Sigma$. We also characterise the equality case.

Theorem A. Let $\left(M^{3}, g\right)$ be a Riemannian 3-manifold with nonempty boundary, and assume that $R_{M}$ and $H_{\partial M}$ are bounded from below. If $\Sigma$ is a compact two-sided capillary stable minimal surface, immersed in $M$ with contact angle $\theta \in(0, \pi)$, then

$$
\mathrm{I}_{\theta}(\Sigma) \leq 2 \pi \chi(\Sigma)
$$

where $\chi(\Sigma)$ denotes the Euler characteristic of $\Sigma$. Moreover, equality occurs if and only if $\Sigma$ satisfies the following properties:

(i) $\Sigma$ is totally geodesic in $M$ and the geodesic curvature of $\partial \Sigma$ in $\partial M$ is equal to $(\cot \theta)$ inf $H_{\partial M}$;

(ii) the scalar curvature $R_{M}$ is constant along $\Sigma$ and equal to inf $R_{M}$, and the mean curvature $H_{\partial M}$ is constant along $\partial \Sigma$ and equal to inf $H_{\partial M}$;

(iii) $\operatorname{Ric}(N)=0$ and $\mathbb{I}(\bar{\nu}, \bar{\nu})=0$. 
In particular, (i), (ii) and (iii) imply that $\Sigma$ has constant Gaussian curvature equal to inf $R_{M} / 2$ and $\partial \Sigma$ has constant geodesic curvature equal to $\inf H_{\partial M} / \sin \theta$.

We begin with a simple lemma.

Lemma 3.2.1. Let $\left(M^{3}, g\right)$ be a Riemannian 3-manifold with nonempty boundary, and let $\Sigma$ be a two-sided capillary CMC surface embedded in $M$ with contact angle $\theta \in(0, \pi)$ and mean curvature equal to $H$. Then:

$$
\mathbb{I}(\bar{\nu}, \bar{\nu})+(\cos \theta) A(\nu, \nu)+(\sin \theta) k_{g}=H_{\partial M}+H \cos \theta
$$

Proof. Let $T$ be a unit tangent vector field along $\partial \Sigma$. Since $\Sigma$ is CMC, we know that $A(\nu, \nu)=$ $H-A(T, T)$. So,

$$
\begin{aligned}
(\cos \theta) A(\nu, \nu)+(\sin \theta) k_{g} & =H \cos \theta-(\cos \theta) A(T, T)+(\sin \theta) k_{g} \\
& =H \cos \theta+g\left(-\nabla_{T} T,(\cos \theta) N+(\sin \theta) \nu\right) \\
& =H \cos \theta+g\left(-\nabla_{T} T, \bar{N}\right) \\
& =H \cos \theta+\mathbb{I}(T, T) .
\end{aligned}
$$

But $\{T, \bar{\nu}\}$ is an orthonormal referential for $T(\partial M)$ along $\partial \Sigma$. Thus,

$$
\mathbb{I}(\bar{\nu}, \bar{\nu})+(\cos \theta) A(\nu, \nu)+(\sin \theta) k_{g}=\mathbb{I}(\bar{\nu}, \bar{\nu})+\mathbb{I}(T, T)+H \cos \theta=H_{\partial M}+H \cos \theta,
$$

as we wanted.

Proof of Theorem A. Since $\Sigma$ is capillary minimal stable, we know that $Q(f, f) \geq 0$ for any $f \in$ $H^{1}(\Sigma)$, where $Q$ is the index form given by (3.1). In particular, taking $f \equiv 1$ yields

$$
\int_{\Sigma}\left(\operatorname{Ric}(N)+\|A\|^{2}\right) \mathrm{d} A+\int_{\partial \Sigma} q \mathrm{~d} L \leq 0
$$

By the Gauss equation, we have:

$$
\operatorname{Ric}(N)=\frac{1}{2}\left(R_{M}+H_{\Sigma}^{2}-\|A\|^{2}\right)-K_{\Sigma},
$$

where $H_{\Sigma} \equiv 0$ and $K_{\Sigma}$ are the mean and Gaussian curvatures of $\Sigma$. Plugging this into (3.3) and using Gauss-Bonnet theorem for the term involving $K_{\Sigma}$, we obtain:

$$
\begin{aligned}
0 & \geq \frac{1}{2} \int_{\Sigma}\left(R_{M}+\|A\|^{2}\right) \mathrm{d} A-\left(2 \pi \chi(\Sigma)-\int_{\partial \Sigma} k_{g} \mathrm{~d} L\right)+\frac{1}{\sin \theta} \int_{\partial \Sigma}[\mathbb{I}(\bar{\nu}, \bar{\nu})+(\cot \theta) A(\nu, \nu)] \mathrm{d} L \\
& =\frac{1}{2} \int_{\Sigma}\left(R_{M}+\|A\|^{2}\right) \mathrm{d} A+\frac{1}{\sin \theta} \int_{\partial \Sigma}\left[\mathbb{I}(\bar{\nu}, \bar{\nu})+(\cot \theta) A(\nu, \nu)+(\sin \theta) k_{g}\right] \mathrm{d} L-2 \pi \chi(\Sigma) \\
& \geq \frac{1}{2} \int_{\Sigma} R_{M} \mathrm{~d} A+\frac{1}{\sin \theta} \int_{\partial \Sigma}\left[\mathbb{I}(\bar{\nu}, \bar{\nu})+(\cot \theta) A(\nu, \nu)+(\sin \theta) k_{g}\right] \mathrm{d} L-2 \pi \chi(\Sigma) .
\end{aligned}
$$

Using Lemma 3.2.1 with $H=0$, we have:

$$
\begin{aligned}
0 & \geq \frac{1}{2} \int_{\Sigma} R_{M} \mathrm{~d} A+\frac{1}{\sin \theta} \int_{\partial \Sigma} H_{\partial M} \mathrm{~d} L-2 \pi \chi(\Sigma) \\
& \geq \frac{1}{2} \inf _{M} R_{M}|\Sigma|+\frac{1}{\sin \theta} \inf _{\partial M} H_{\partial M}|\partial \Sigma|-2 \pi \chi(\Sigma),
\end{aligned}
$$

proving the first assertion of the theorem.

Let us assume now that equality holds. It is immediate to see that $\Sigma$ must be totally geodesic, item (b) holds and $Q(1,1)=0$. The latter implies that $Q(1, f)=0$ for any $f \in H^{1}(\Sigma)$. Indeed, if 
$c>0$, then

$$
0 \leq Q(1-c f, 1-c f)=Q(1,1)-2 c Q(1, f)+c^{2} Q(f, f)=-2 c Q(1, f)+c^{2} Q(f, f) .
$$

Dividing by $c$ and rearranging, we obtain

$$
Q(1, f) \leq \frac{c}{2} Q(f, f)
$$

Now we let $c \searrow 0$ and conclude that $Q(1, f) \leq 0$. The reverse inequality is analogous. This proves the claim. Thus, we know that

$$
Q(1, f)=\int_{\Sigma} \operatorname{Ric}(N) f \mathrm{~d} A+\int_{\partial \Sigma} q f \mathrm{~d} L=0
$$

for any $f \in H^{1}(\Sigma)$. This implies that $\operatorname{Ric}(N)=0$ and $q=0$. Since we already know that $A=0$, it follows that $\mathbb{I}(\bar{\nu}, \bar{\nu})=0$, proving item (c).

It remains to calculate the geodesic curvature $\bar{k}_{g}$ of $\Sigma$ in $\partial M$. We have

$$
\bar{k}_{g}:=g\left(-\nabla_{T} T, \bar{\nu}\right)=g\left(-\nabla_{T} T,-(\sin \theta) N+(\cos \theta) \nu\right)=-(\sin \theta) A(T, T)+(\cos \theta) k_{g}=(\cos \theta) k_{g} .
$$

But Lemma 3.2.1 implies that $(\sin \theta) k_{g}=H_{\partial M}$. So, $\bar{k}_{g}=(\cot \theta) H_{\partial M}$, proving (a). The converse statement can be easily proved.

Given a properly embedded and infinitesimally rigid surface $\Sigma$ in $M$, Proposition 3.1.2 furnishes a vector field $Z$ in $\Sigma$ such that $g(Z, N)=1$ and $Z$ is tangent to $\partial M$ along $\partial \Sigma$ (just take $f \equiv 1$ and define $Z=\xi_{\Phi}$ ). Let us also denote by $Z$ an extension to $M$ which is tangent to $\partial M$ along the entire boundary of $M$. Let $\phi=\phi(x, t)$ the local flow of $Z$ and fix a number $\alpha$ between 0 and 1 . We are amost ready to prove Theorem $B$. The proof is an adaptation of the one given by Ambrozio in [Amb15].

Theorem B. Let $\left(M^{3}, g\right)$ be a Riemannian 3-manifold with nonempty boundary, and assume that $R_{M}$ and $H_{\partial M}$ are bounded from below. Let $\Sigma$ be a compact two-sided capillary minimal surface, properly embedded in $M$ with contact angle $\theta \in(0, \pi)$. If $\Sigma$ is infinitesimally rigid, then there exists $\varepsilon>0$ and a map $w \in C^{2, \alpha}(\Sigma \times(-\varepsilon, \varepsilon))$ such that for every $t \in(-\varepsilon, \varepsilon)$, the set

$$
\Sigma_{t}=\{\phi(x, w(x, t)): x \in \Sigma\}
$$

is a capillary $C M C$ surface with contact angle $\theta$ and mean curvature $H(t)$. Moreover, for each $x \in \Sigma$ and $t \in(-\varepsilon, \varepsilon)$,

$$
w(x, 0)=0, \quad \int_{\Sigma}(w(\cdot, t)-t) \mathrm{d} A=0, \quad \text { and } \quad \frac{\partial w}{\partial t}(x, 0)=1 .
$$

In particular, taking a smaller $\varepsilon>0$ if necessary, $\left\{\Sigma_{t}\right\}_{t \in(-\varepsilon, \varepsilon)}$ is a capillary CMC foliation of a neighbourhood of $\Sigma$ in $M$.

We begin with a lemma, whose proof can be found in the appendices of [Amb15] and [Li20].

Lemma 3.2.2. Let $\left(M^{3}, g\right)$ be a Riemannian 3-manifold with nonempty boundary, and let $\varphi: \Sigma \rightarrow$ $M$ a two-sided proper embedding of a compact surface $\Sigma$ with boundary. Fix a proper variation $\Phi: \Sigma \times(-\varepsilon, \varepsilon) \rightarrow M$ of $\varphi$ and let $\Sigma_{t}=\Phi(\Sigma \times\{t\})$. We use the subscript $t$ to denote all terms related to $\Sigma_{t}$, e.g. $N_{t}$ is the unit normal, $H_{t}$ is the mean curvature, etc. Let $\rho_{t}=g\left(N_{t}, \xi_{\Phi, t}\right)$ and let 
$\theta_{t}$ be the contact angle between $\Sigma_{t}$ and $\partial M$. Then the following formulae hold:

$$
\begin{aligned}
\frac{\partial H_{t}}{\partial t} & =L_{\Sigma_{t}}\left(\rho_{t}\right)-\mathrm{d} H_{t}\left(\xi_{\Phi, t}^{\top}\right) \quad \text { on } \Sigma_{t}, \\
\frac{\partial\left(\cos \theta_{t}\right)}{\partial \nu_{t}} & =-\left(\sin \theta_{t}\right) \frac{\partial \rho_{t}}{\partial \nu_{t}}+\left(\cos \theta_{t}\right) A_{t}\left(\nu_{t}, \nu_{t}\right) \rho_{t}+\mathbb{I}\left(\bar{\nu}_{t}, \bar{\nu}_{t}\right) \rho_{t}+\mathrm{d} \theta_{t}\left(W_{t}\right) \quad \text { on } \partial \Sigma_{t},
\end{aligned}
$$

where $(\cdot)^{\top}$ denotes the orthogonal projection onto $T \Sigma_{t}, L_{\Sigma_{t}}$ is the Jacobi operator of $\Sigma_{t}$ and $W_{t}$ is a certain vector field along $\partial \Sigma_{t}$. In particular, if each $\Sigma_{t}$ is capillary CMC with contact angle $\theta \in(0, \pi)$, then

$$
\begin{aligned}
\frac{\mathrm{d} H_{t}}{\mathrm{~d} t} & =L_{\Sigma_{t}}\left(\rho_{t}\right) \quad \text { on } \Sigma_{t} \\
\frac{\partial \rho_{t}}{\partial \nu_{t}} & =\left[\frac{1}{\sin \theta} \mathbb{I}\left(\bar{\nu}_{t}, \bar{\nu}_{t}\right)+(\cot \theta) A_{t}\left(\nu_{t}, \nu_{t}\right)\right] \rho_{t} \quad \text { on } \partial \Sigma_{t}
\end{aligned}
$$

Proof of Theorem B. Given a function $u \in C^{2, \alpha}(\Sigma)$, let $\Sigma_{u}=\{\phi(x, u(x)): x \in \Sigma\}$. If the norm of $u$ is small enough, then $\Sigma_{u}$ is a properly embedded surface in $M$. As in the statement of Lemma 3.2.2 we use the subscript $u$ to denote the terms related to $\Sigma_{u}$.

Now consider the Banach spaces

$$
\begin{aligned}
& E=\left\{u \in C^{2, \alpha}(\Sigma): \int_{\Sigma} u \mathrm{~d} A=0\right\}, \\
& F=\left\{u \in C^{0, \alpha}(\Sigma): \int_{\Sigma} u \mathrm{~d} A=0\right\} .
\end{aligned}
$$

For small $\varepsilon>0$ and $\delta>0$, define the map $\Psi: B_{E}(0, \delta) \times(-\varepsilon, \varepsilon) \rightarrow F \times C^{1, \alpha}(\partial \Sigma)$ by

$$
\Psi(u, t)=\left(H_{u+t}-\frac{1}{|\Sigma|} \int_{\Sigma} H_{u+t} \mathrm{~d} A, g\left(N_{u+t}, \bar{N}_{u+t}\right)-\cos \theta\right)
$$

where $B_{E}(0, \delta)$ denotes the open ball of radius $\delta$ centered at the origin of $E$. We claim that $D \Psi(0,0)$ : $E \times \mathbb{R}$ is an isomorphism when restricted to $\{0\} \times E$. To prove this, let $v \in E$. Notice that the map $\Phi: \Sigma \times(-\varepsilon, \varepsilon) \ni(x, s) \mapsto \phi(x, s v(x)) \in M$ is a proper variation of $\Sigma$ whose variational vector field is

$$
\xi_{\Phi}(x)=\left.\frac{\partial}{\partial s}\right|_{s=0} \phi(x, s v(x))=v(x) \frac{\partial \phi}{\partial s}(x, 0)=v(x) Z(x), \quad x \in \Sigma .
$$

So, using the formulae from Lemma 3.2.2 and the fact that the Jacobi operator of $\Sigma$ is just the Laplacian, since $\Sigma$ is infinitesimally rigid, we obtain:

$$
\begin{aligned}
D \Psi(0,0) \cdot(v, 0) & =\left.\frac{\mathrm{d}}{\mathrm{d} s}\right|_{s=0} \Psi(s v, 0)=\left(\Delta_{\Sigma} v-\frac{1}{|\Sigma|} \int_{\Sigma} \Delta_{\Sigma} v \mathrm{~d} A,-(\sin \theta) \frac{\partial v}{\partial \nu}\right) \\
& =\left(\Delta_{\Sigma} v-\frac{1}{|\Sigma|} \int_{\partial \Sigma} \frac{\partial v}{\partial \nu} \mathrm{d} A,-(\sin \theta) \frac{\partial v}{\partial \nu}\right) .
\end{aligned}
$$

If this is equal to zero, then $v$ is harmonic and satisfies a Neumann condition on the boundary of $\Sigma$. This implies that $v$ is constant, and this constant must be equal to zero since $v \in E$, which proves that $D \Psi(0,0)$ is injective. The surjectivity follows from classical results for Neumann type boundary conditions for the Laplace operator.

The final step is to apply the implicit function theorem: for some smaller $\varepsilon>0$, there exists a function $(-\varepsilon, \varepsilon) \ni t \mapsto u(t) \in B_{E}(0, \delta)$ such that $u(0)=0$ and $\Psi(u(t), t)=(0,0)$ for any $t \in(-\varepsilon, \varepsilon)$. 
This means precisely that the surfaces

$$
\Sigma_{u(t)+t}=\{\phi(x, u(t)(x)+t): x \in \Sigma\}
$$

have constant mean curvature and meet $\partial M$ along their boundaries at an angle of $\theta$.

Now let $w: \Sigma \times(-\varepsilon, \varepsilon) \rightarrow \mathbb{R}$ be given by $w(x, t)=u(t)(x)+t$. Then $w(x, 0)=0$ and $w(\cdot, t)-t=$ $u(\cdot) \in B_{E}(0, \delta)$. Define $G: \Sigma \times(-\varepsilon, \varepsilon) \rightarrow M$ by $G(x, t)=\phi(x, w(x, t))$ and observe that this is a proper variation of $\Sigma$ whose variational vector field is

$$
\xi_{G}(x)=\frac{\partial G}{\partial t}(x, 0)=\frac{\partial w}{\partial t}(x, 0) \frac{\partial \phi}{\partial t}(x, 0)=\frac{\partial w}{\partial t}(x, 0) Z(x), \quad x \in \Sigma .
$$

Since

$$
0=\Psi(u(t), t)=\left(H_{w(\cdot, t)}-\frac{1}{|\Sigma|} \int_{\Sigma} H_{w(\cdot, t)} \mathrm{d} A, g\left(N_{w(\cdot, t)}, \bar{N}_{w(\cdot, t)}\right)-\cos \theta\right)
$$

for all $t \in(-\varepsilon, \varepsilon)$, differentiating this equation with respect to $t$ implies that $\left.\frac{\partial w}{\partial t}\right|_{t=0}$ satisfies the Neumann problem on $\Sigma$ (see equation (3.6)). Therefore, it must be constant. But

$$
\int_{\Sigma}(w(x, t)-t) \mathrm{d} A(x)=\int_{\Sigma} u(t) \mathrm{d} A=0, \quad t \in(-\varepsilon, \varepsilon)
$$

since $u(t) \in E$. By differentiating this equation with respect to $t$ and evaluating it at $t=0$ we obtain that $\left.\int_{\Sigma} \frac{\partial w}{\partial t}\right|_{t=0} \mathrm{~d} A=|\Sigma|$. Thus, $\left.\frac{\partial w}{\partial t}\right|_{t=0}=1$ as we wanted to show.

Finally, since $G(x, 0)=\phi(x, 0)=x$ for every $x \in \Sigma, \xi_{G}=Z$ is transverse to $\Sigma$ and $\Sigma$ is properly embedded, by taking a smaller $\varepsilon>0$ if necessary, we may assume that $G$ parametrises a foliation of a neighbourhood of $\Sigma$ in $M$. This concludes the proof.

The proof of Theorem C is similar to the proof of Theorem B. This way, we only sketch it, indicating the main differences.

Theorem C. Let $\left(M^{3}, g\right)$ be a Riemannian 3-manifold with nonempty boundary, and assume that $R_{M}$ and $H_{\partial M}$ are bounded from below. Let $\Sigma$ be a compact two-sided capillary minimal surface, properly embedded in $M$ with contact angle $\theta \in(0, \pi)$. If $\Sigma$ is infinitesimally rigid, then there exists $\varepsilon>0$ and a map $w \in C^{2, \alpha}(\Sigma \times(-\varepsilon, \varepsilon))$ such that for every $t \in(-\varepsilon, \varepsilon)$, the set

$$
\Sigma_{t}=\{\phi(x, w(x, t)): x \in \Sigma\}
$$

is a capillary minimal surface with contact angle $\theta(t) \in(0, \pi)$. Moreover, for each $x \in \Sigma$ and $t \in(-\varepsilon, \varepsilon)$,

$$
w(x, 0)=0, \quad \int_{\Sigma}(w(\cdot, t)-t) \mathrm{d} A=0, \quad \text { and } \quad \frac{\partial w}{\partial t}(x, 0)=1 .
$$

In particular, taking a smaller $\varepsilon>0$ if necessary, $\left\{\Sigma_{t}\right\}_{t \in(-\varepsilon, \varepsilon)}$ is a capillary minimal foliation of a neighbourhood of $\Sigma$ in $M$.

Proof of Theorem $C$. As in the previous proof, for $u \in C^{2, \alpha}(\Sigma)$, let $\Sigma_{u}=\{\phi(x, u(x)): x \in \Sigma\}$.

Now consider the Banach spaces

$$
\begin{aligned}
& E=\left\{u \in C^{2, \alpha}(\Sigma): \int_{\Sigma} u \mathrm{~d} A=0\right\}, \\
& G=\left\{u \in C^{1, \alpha}(\partial \Sigma): \int_{\partial \Sigma} u \mathrm{~d} L=0\right\} .
\end{aligned}
$$


For small $\varepsilon>0$ and $\delta>0$, define the map $\Lambda: B_{E}(0, \delta) \times(-\varepsilon, \varepsilon) \rightarrow G \times C^{0, \alpha}(\Sigma)$ by

$$
\Lambda(u, t)=\left(g\left(N_{u+t}, \bar{N}_{u+t}\right)-\frac{1}{|\partial \Sigma|} \int_{\partial \Sigma} g\left(N_{u+t}, \bar{N}_{u+t}\right) \mathrm{d} L, H_{u+t}\right),
$$

where $B_{E}(0, \delta)$ denotes the open ball of radius $\delta$ centered at the origin of $E$. Then, using the formulae from Lemma 3.2.2 and the fact that the Jacobi operator of $\Sigma$ is just the Laplacian, since $\Sigma$ is infinitesimally rigid, we have:

$$
D \Lambda(0,0) \cdot(v, 0)=\left.\frac{\mathrm{d}}{\mathrm{d} s}\right|_{s=0} \Psi(s v, 0)=\left(-(\sin \theta) \frac{\partial v}{\partial \nu}+\frac{\sin \theta}{|\partial \Sigma|} \int_{\partial \Sigma} \frac{\partial v}{\partial \nu} \mathrm{d} L, \Delta_{\Sigma} v\right)
$$

for any $v \in E$. It is easy to show that $D \Lambda(0,0)$ is an isomorphism when restricted to $E \times\{0\}$. Now, just apply the implicit function theorem and proceed as in the proof of Theorem B.

Before proving Theorem $\mathrm{D}$, let us clarify that a closed curve $\gamma$ in $\partial M$ is locally length-minimising when every nearby closed curve in $\partial M$ has length greater than or equal to the length of $\gamma$.

Theorem D. Let $\left(M^{3}, g\right)$ be a Riemannian 3-manifold with nonempty and weakly mean-convex boundary, and assume that $R_{M}$ is bounded from below. Let $\Sigma$ be an energy-minimising and infinitesimally rigid surface, properly embedded in $M$ with contact angle $\theta \in(0, \pi)$. Assume that one of the following hypotheses holds:

(a) each component of $\partial \Sigma$ is locally length-minimising in $\partial M$; or

(b) $\inf _{\partial M} H_{\partial M}=0$.

Then either $\theta=\pi / 2$ or $\Sigma$ is a flat and totally geodesic cylinder, $M$ is flat and $\partial M$ is totally geodesic around $\Sigma$. In the first case, there is a neighbourhood of $\Sigma$ in $M$ that is isometric to $(\Sigma \times$ $\left.(-\varepsilon, \varepsilon), g_{\Sigma}+\mathrm{d} t^{2}\right)$, where $\left(\Sigma, g_{\Sigma}\right)$ has constant Gaussian curvature $\inf R_{M} / 2$ and $\partial \Sigma$ has constant geodesic curvature inf $H_{\partial M}$ in $\Sigma$.

Let us begin with a lemma.

Lemma 3.2.3. Let $\left(M^{3}, g\right)$ be a Riemannian 3-manifold with nonempty boundary and let $\varphi: \Sigma \rightarrow$ $M$ be a two-sided capillary minimal proper immersion of a compact surface $\Sigma$, with contact angle equal to $\theta \in(0, \pi)$. If $\varphi$ is energy-minimising for the angle $\theta$, then, changing the sign of its unit normal, it is energy-minimising for the angle $\pi-\theta$.

Proof. Given a proper variation $\Phi: \Sigma \times(-\varepsilon, \varepsilon) \rightarrow M$ of $\varphi$, define $\tilde{\Phi}: \Sigma \times(-\varepsilon, \varepsilon) \rightarrow M$ to be the variation given by $\tilde{\Phi}(x, t)=\Phi(x,-t)$. Denote by $W_{\Phi}$ and $W_{\tilde{\Phi}}$ the wetting areas of $\Phi$ and $\tilde{\Phi}$, respectively. We claim the $W_{\tilde{\Phi}}(t)=-W_{\Phi}(-t)$ for every $t \in(-\varepsilon, \varepsilon)$. To prove this, define $f: \partial \Sigma \times(-\varepsilon, \varepsilon)$ by

$$
f=\Phi^{*}\left(\mathrm{~d} A_{\partial M}\right)(T, \mathrm{~d} s),
$$

where $T$ is a unit tangent vector field along $\partial \Sigma$ and $\mathrm{d} s$ is the standard vector field in $\mathbb{R}$. Then, for $t>0$, we have

$$
\begin{aligned}
W_{\tilde{\Phi}}(t) & =\int_{\partial \Sigma \times[0, t]} f(x,-s) \mathrm{d} V(x, s)=\int_{\partial \Sigma} \int_{0}^{t} f(x,-s) \mathrm{d} s \mathrm{~d} x \\
& =-\int_{\partial \Sigma} \int_{0}^{-t} f(x, u) \mathrm{d} u \mathrm{~d} x \\
& =-\int_{\partial \Sigma \times[0,-t]} f(x, u) \mathrm{d} V(x, u) \\
& =-W_{\Phi}(-t) .
\end{aligned}
$$


The computation is analogous for $t<0$. This proves the claim. To prove the lemma, we show that $E_{\tilde{\Phi}, \pi-\theta}(t)$ is equal to $E_{\Phi, \theta}(-t)$ :

$$
\begin{aligned}
E_{\tilde{\Phi}, \pi-\theta}(t) & =A(\tilde{\Phi}(\Sigma, t))-(\cos (\pi-\theta)) W_{\tilde{\Phi}}(t) \\
& =A(\Phi(\Sigma,-t))-(\cos \theta) W_{\Phi}(-t) \\
& =E_{\Phi, \theta}(-t) .
\end{aligned}
$$

Thus, we have

$$
E_{\tilde{\Phi}, \pi-\theta}(t)=E_{\Phi, \theta}(-t) \geq E_{\Phi, \theta}(0)=E_{\tilde{\Phi}, \pi-\theta}(0)
$$

for any variation $\Phi$ and any $t$, which implies that $\varphi$ is energy-minimising for the angle $\pi-\theta$.

Proof of Theorem D. Let $G$ be a parametrisation of the capillary CMC foliation $\left\{\Sigma_{t}\right\}_{t \in(-\varepsilon, \varepsilon)}$ given by Theorem B. For each $t \in(-\varepsilon, \varepsilon)$, let $\rho_{t}=g\left(\partial_{G}, N_{t}\right)$, where as usual, we use the subscript $t$ for terms related to $\Sigma_{t}$. Then, by equations (3.4) and (3.5) in Lemma 3.2.2, we have

$$
\begin{aligned}
H^{\prime}(t) & =\Delta_{t} \rho_{t}+\left(\operatorname{Ric}\left(N_{t}\right)+\left\|A_{t}\right\|^{2}\right) \rho_{t} \quad \text { on } \Sigma_{t}, \\
\frac{\partial \rho_{t}}{\partial \nu_{t}} & =q_{t} \rho_{t}=\left[\frac{1}{\sin \theta} \mathbb{I}\left(\bar{\nu}_{t}, \bar{\nu}_{t}\right)+(\cot \theta) A_{t}\left(\nu_{t}, \nu_{t}\right)\right] \rho_{t} \quad \text { on } \partial \Sigma_{t} .
\end{aligned}
$$

Notice that $\rho_{0}=g(Z, N)=1$, so we can assume $\rho_{t}>0$ for any $t \in(-\varepsilon, \varepsilon)$, maybe for some smaller $\varepsilon>0$. Using the Gauss equation, we rewrite equation (3.9) as

$$
H^{\prime}(t) \rho_{t}^{-1}=\rho_{t}^{-1} \Delta_{t} \rho_{t}+\frac{1}{2}\left(R_{M, t}+H(t)^{2}+\left\|A_{t}\right\|^{2}\right)-K_{t},
$$

where $R_{M, t}$ denotes the scalar curvature of $M$ restricted to $\Sigma_{t}$ and $K_{t}$ denotes the Gaussian curvature of $\Sigma_{t}$. Now recall that $H(t)$ is constant on $\Sigma_{t}$, so, integration by parts gives

$$
\begin{aligned}
H^{\prime}(t) \int_{\Sigma} \rho_{t}^{-1} \mathrm{~d} A_{t} & =\int_{\Sigma} \rho_{t}^{-2}\left\|\nabla \rho_{t}\right\|^{2} \mathrm{~d} A_{t}+\int_{\partial \Sigma} \rho_{t}^{-1} \frac{\partial \rho_{t}}{\partial \nu_{t}} \mathrm{~d} L_{t}+\frac{1}{2} \int_{\Sigma}\left(R_{M, t}+H(t)^{2}+\left\|A_{t}\right\|^{2}-2 K_{t}\right) \mathrm{d} A_{t} \\
& =\int_{\Sigma} \rho_{t}^{-2}\left\|\nabla \rho_{t}\right\|^{2} \mathrm{~d} A_{t}+\int_{\partial \Sigma} q_{t} \mathrm{~d} L_{t}+\frac{1}{2} \int_{\Sigma}\left(R_{M, t}+H(t)^{2}+\left\|A_{t}\right\|^{2}\right) \mathrm{d} A_{t}-\int_{\Sigma} K_{t} \mathrm{~d} A_{t} \\
& \geq \int_{\partial \Sigma} q_{t} \mathrm{~d} L_{t}+\frac{1}{2} \int_{\Sigma}\left(R_{M, t}+H(t)^{2}+\left\|A_{t}\right\|^{2}\right) \mathrm{d} A_{t}-\int_{\Sigma} K_{t} \mathrm{~d} A_{t} .
\end{aligned}
$$

We now apply Gauss-Bonnet Theorem and Lemma 3.2.1 to obtain

$$
\begin{aligned}
H^{\prime}(t) \int_{\Sigma} \rho_{t}^{-1} \mathrm{~d} A_{t} & \geq \int_{\partial \Sigma}\left(q_{t}+k_{g, t}\right) \mathrm{d} L_{t}+\frac{1}{2} \int_{\Sigma}\left(R_{M, t}+H(t)^{2}+\left\|A_{t}\right\|^{2}\right) \mathrm{d} A_{t}-2 \pi \chi\left(\Sigma_{t}\right) \\
& =\frac{1}{\sin \theta} \int_{\partial \Sigma}\left(H_{\partial M, t}+H(t) \cos \theta\right) \mathrm{d} L_{t}+\frac{1}{2} \int_{\Sigma}\left(R_{M, t}+H(t)^{2}+\left\|A_{t}\right\|^{2}\right) \mathrm{d} A_{t}-2 \pi \chi\left(\Sigma_{t}\right) \\
& \geq \frac{1}{2} \int_{\Sigma} R_{M, t} \mathrm{~d} A_{t}+\frac{1}{\sin \theta} \int_{\partial \Sigma} H_{\partial M, t} \mathrm{~d} L_{t}+H(t)\left|\partial \Sigma_{t}\right| \cot \theta-2 \pi \chi\left(\Sigma_{t}\right) .
\end{aligned}
$$

Since each $\Sigma_{t}$ is homeomorphic to $\Sigma, \chi\left(\Sigma_{t}\right)=\chi(\Sigma)$. Next, we use the fact that $\Sigma$ is infinitesimally rigid to apply the identity $\mathrm{I}_{\theta}(\Sigma)=2 \pi \chi(\Sigma)$ :

$$
\begin{aligned}
H^{\prime}(t) \int_{\Sigma} \rho_{t}^{-1} \mathrm{~d} A_{t} & \geq \frac{1}{2} \inf _{M} R_{M}\left|\Sigma_{t}\right|+\frac{1}{\sin \theta} \inf _{\partial \Sigma} H_{\partial M}\left|\partial \Sigma_{t}\right|+H(t)\left|\partial \Sigma_{t}\right| \cot \theta-2 \pi \chi\left(\Sigma_{t}\right) \\
& =\frac{1}{2} \inf _{M} R_{M}\left(\left|\Sigma_{t}\right|-|\Sigma|\right)+\frac{1}{\sin \theta} \inf _{\partial \Sigma} H_{\partial M}\left(\left|\partial \Sigma_{t}\right|-|\partial \Sigma|\right)+H(t)\left|\partial \Sigma_{t}\right| \cot \theta .
\end{aligned}
$$

By hypothesis, inf $H_{\partial M} \geq 0$. If each component of $\partial \Sigma$ is length-minimising, then the second term 
in the right hand side is nonnegative, and if inf $H_{\partial M}=0$, then it is equal to zero. In either case, we have

$$
\begin{aligned}
H^{\prime}(t) \int_{\Sigma} \rho_{t}^{-1} \mathrm{~d} A & \geq \frac{1}{2} \inf _{M} R_{M}\left(\left|\Sigma_{t}\right|-|\Sigma|\right)+H(t)\left|\partial \Sigma_{t}\right| \cot \theta \\
& =\frac{1}{2} \inf _{M} R_{M} \int_{0}^{t} \frac{\mathrm{d}}{\mathrm{d} s}\left|\Sigma_{s}\right| \mathrm{d} s+H(t)\left|\partial \Sigma_{t}\right| \cot \theta
\end{aligned}
$$

By the first variation of area,

$$
\begin{aligned}
\frac{\mathrm{d}}{\mathrm{d} s}\left|\Sigma_{s}\right| & =-\int_{\Sigma} H(s) \rho_{s} \mathrm{~d} A_{s}+\int_{\partial \Sigma} g\left(\nu_{s}, \partial_{t} G\right) \mathrm{d} L_{s} \\
& =-H(s) \int_{\Sigma} \rho_{s} \mathrm{~d} A_{s}-\cot \theta \int_{\partial \Sigma} \rho_{s} \mathrm{~d} L_{s} .
\end{aligned}
$$

Thus, we obtain the following differential inequality:

$$
H^{\prime}(t) \int_{\Sigma} \rho_{t}^{-1} \mathrm{~d} A \geq-\frac{1}{2} \inf _{M} R_{M} \int_{0}^{t}\left[H(s) \int_{\Sigma} \rho_{s} \mathrm{~d} A_{s}+\cot \theta \int_{\partial \Sigma} \rho_{s} \mathrm{~d} L_{s}\right] \mathrm{d} s+H(t)\left|\partial \Sigma_{t}\right| \cot \theta
$$

Let us rewrite this as

$$
H^{\prime}(t) \geq \frac{R_{0}}{\psi(t)} \int_{0}^{t} H(s) \xi(s) \mathrm{d} s+\cot \theta\left[H(t)\left|\partial \Sigma_{t}\right|+\frac{R_{0}}{\psi(t)} \int_{0}^{t} \eta(s) \mathrm{d} s\right]
$$

where

- $R_{0}=-\frac{1}{2} \inf _{M} R_{M}$

- $\psi(t)=\int_{\Sigma} \rho_{t}^{-1} \mathrm{~d} A_{t}$

- $\xi(t)=\int_{\Sigma} \rho_{t} \mathrm{~d} A_{t}$

- $\eta(t)=\int_{\partial \Sigma} \rho_{t} \mathrm{~d} L_{t}$

It is time to apply Lemma 3.2.3. Changing the sign of the unit normal $N$ of $\Sigma$ in the begining of the proof, if necessary, we may assume that $\theta \in(0, \pi / 2]$ if $R_{0} \geq 0$ and that $\theta \in[\pi / 2, \pi)$ if $R_{0}<0$. In either case, inequality (3.12) implies that $H^{\prime}(0) \geq 0$. If $H^{\prime}(0)>0$ then $H(t)>0$ for all small positive $t$. The first variation formula for the energy (see Proposition 3.1.1) would then give that $E(t)<E(0)$ :

$$
E(t)-E(0)=\int_{0}^{t} E^{\prime}(s) \mathrm{d} s=-\int_{0}^{t} H(s)\left(\int_{\Sigma} \rho_{s} \mathrm{~d} A_{s}\right) \mathrm{d} s<0,
$$

which is absurd, since $\Sigma$ is energy-minimising. So $H^{\prime}(0)=0$. Now let $\alpha(t)$ denote the real function in the right hand side of inequality (3.12). A simple computation yields $\alpha^{\prime}(0)=\frac{\eta(0)}{\psi(0)} R_{0} \cot \theta$. This is positive if and only if $R_{0} \neq 0$ and $\theta \neq \pi / 2$. In this case, we would have $H^{\prime \prime}(0) \geq \alpha^{\prime}(0)>0$, which is also absurd by the same reason. So either $\theta=\pi / 2$, and we are done, or $R_{0}=0$.

Let us analyse the case $R_{0}=0$. Inequality (3.12) takes the form $H^{\prime}(t) \geq(\cot \theta)\left|\partial \Sigma_{t}\right| H(t)$, and since $\cot \theta \geq 0$, this implies that $H(t) \geq 0$ for $t \geq 0$ and $H(t) \leq 0$ for $t \leq 0$. By the first variation of energy again, $E(t) \leq E(0)$ for any $t \in(-\varepsilon, \varepsilon)$. Thus, $E(t) \equiv E(0)$ because $\Sigma$ is energy-minimising. So, $H(t) \equiv 0$ by the same formula. In particular, every $\Sigma_{t}$ is capillary minimal stable. Applying Theorem A we obtain that, for every $t$,

$$
2 \pi \chi\left(\Sigma_{t}\right) \geq \mathrm{I}_{\theta}\left(\Sigma_{t}\right)=\frac{1}{\sin \theta} \inf _{\partial M} H_{\partial M}\left|\partial \Sigma_{t}\right| \geq \frac{1}{\sin \theta} \inf _{\partial M} H_{\partial M}|\partial \Sigma|=\mathrm{I}_{\theta}(\Sigma)=2 \pi \chi(\Sigma)=2 \pi \chi\left(\Sigma_{t}\right) .
$$


This way, every inequality above is an equality, which shows that every $\Sigma_{t}$ is infinitesimally rigid. In particular, $\partial \Sigma_{t}$ has constant geodesic curvature equal to $(\cot \theta) \inf _{\partial M} H_{\partial M}$ in $\partial M$. Since we are supposing either condition (a) or (b) in the theorem, we conclude that either inf $\partial{ }_{\partial M} H_{\partial M}=0$ or each component of $\partial \Sigma$ is a geodesic in $\partial M$. Hence, either $\theta=\pi / 2$ and we are done, $\operatorname{or} \inf _{\partial M} H_{\partial M}=0$.

Assuming $R_{0}=0$ and $\inf _{\partial M} H_{\partial M}=0$ we are going to show that $M$ is flat in a neighbourhood of $\Sigma$ and that $\partial M$ is totally geodesic in this neighbourhood.

Claim: The unit normals $N_{t}$ to $\Sigma_{t}$ define a parallel vector field.

Firstly, notice that each map $\rho_{t}$ is constant because it harmonic with Neumann boundary condition by equations (3.9) and (3.10). Now, let $\left(x_{1}, x_{2}\right)$ be local coordinates on $\Sigma$ and write $E_{i}=\partial_{x_{i}} G$. Since each $\Sigma_{t}$ is totally geodesic, we know that $\nabla_{E_{i}} N_{t}=0$. Moreover, since $N_{t}$ is of unit length, $\nabla_{\partial_{t} G} N_{t}$ is tangent to $\Sigma_{t}$. Thus,

$$
\begin{aligned}
g\left(\nabla_{\partial_{t} G} N_{t}, E_{i}\right) & =\partial_{t} g\left(N_{t}, E_{i}\right)-g\left(N_{t}, \nabla_{\partial_{t} G} E_{i}\right) \\
& =-g\left(N_{t}, \nabla_{E_{i}} \partial_{t} G\right) \\
& =-\partial_{x_{i}} g\left(N_{t}, \partial_{t} G\right)+g\left(\nabla_{E_{i}} N_{t}, \partial_{t} G\right) \\
& =-\partial_{x_{i}} \rho_{t} \\
& =0 .
\end{aligned}
$$

This proves the Claim.

The Gauss equation for $\Sigma_{t}$ shows that $\bar{K}\left(\partial_{x_{1}} G \wedge \partial_{x_{2}} G\right)=K\left(\partial_{x_{1}} G \wedge \partial_{x_{2}} G\right)=0$, where $K$ and $\bar{K}$ denote the sectional curvatures of $\Sigma$ and of $M$. This holds since $\Sigma_{t}$ is flat and totally geodesic. Now, since $N_{t}$ is parallel, $g\left(R(\cdot, \cdot) N_{t}, \cdot\right)=0$. The symmetries of the curvature tensor then imply that $R \equiv 0$, that is, $M$ is flat around $\Sigma$.

Finally, let us show that $\partial M$ is totally geodesic near $\Sigma$. By infinitesimal rigidity we know that $\mathbb{I}\left(\nu_{t}, \nu_{t}\right) \equiv 0$ for every $t \in(-\varepsilon, \varepsilon)$. If we denote by $T_{t}$ a unit normal for $\partial \Sigma_{t}$, it is straighforward to check that $\mathbb{I}\left(T_{t}, \nu_{t}\right) \equiv 0$ and $\mathbb{I}\left(T_{t}, T_{t}\right) \equiv 0$. Since $\left\{\nu_{t}, T_{t}\right\}$ is an orthonormal referential for $T(\partial M)$ around $\partial \Sigma$, the bilinearity of $\mathbb{I}$ implies that $\mathbb{I} \equiv 0$, i.e. $\partial M$ is totally geodesic in the union of all $\partial \Sigma_{t}, t \in(-\varepsilon, \varepsilon)$.

The last statement of the theorem (when $\theta=\pi / 2$ ) follows from Theorem 7 in [Amb15].

Remark 3.2.1. Theorem $D$ can be proved using the capillary minimal foliation with variable contact angle given by Theorem $C$ instead of the capillary CMC foliation of Theorem B.

Finally, we move on to Theorem E, which gives conditions under which we can control the topology of low index surfaces in some Riemannian 3-manifolds.

Theorem E. Let $\left(M^{3}, g\right)$ be a Riemannian 3-manifold with nonempty boundary. Suppose that $\Sigma$ is a compact orientable two-sided capillary minimal surface of genus $g$ and with $k \geq 1$ boundary components, immersed in $M$ with contact angle $\theta \in(0, \pi)$.

(i) Suppose that $(M, g)$ has nonnegative Ricci curvature and weakly mean-convex boundary. If $\Sigma$ has index 1 then

$$
\int_{\partial \Sigma} k_{g} \mathrm{~d} L<2 \pi\left[9-(-1)^{g}-2(g+k)\right]
$$

In particular, if the total geodesic curvature of $\partial \Sigma$ (in $\Sigma$ ) is nonnegative (which happens if $\Sigma$ is free boundary and $\partial M$ is weakly convex, for instance), then

(a) $g+k \leq 3$ if $g$ is even;

(b) $g+k \leq 4$ if $g$ is odd.

(ii) Suppose that the scalar curvature of $(M, g)$ and the mean curvature of $\partial M$ are bounded from 
below. If $\Sigma$ has index 1 , then

$$
\mathrm{I}_{\theta}(\Sigma)=\frac{1}{2} \inf _{M} R_{M}|\Sigma|+\frac{1}{\sin \theta} \inf _{\partial M} H_{\partial M}|\partial \Sigma|<2 \pi\left[7-(-1)^{g}-k\right] .
$$

(iii) Suppose that $(M, g)$ has scalar curvature $R_{M} \geq R_{0}>0$ and weakly mean-convex boundary.

(a) If $\Sigma$ is stable, then it is a disc and $|\Sigma| \leq \frac{4 \pi}{R_{0}}$.

(b) If $\Sigma$ has index 1 , then $|\Sigma| \leq \frac{4 \pi\left[7-(-1)^{g}-k\right]}{R_{0}}$.

As usual, we start with a lemma, whose proof can be found in [FP14].

Lemma 3.2.4. Let $\bar{\Sigma}$ be a closed orientable Riemannian surface of genus $g \geq 0$, and let $h: \bar{\Sigma} \rightarrow \mathbb{R}$ be any nonnegative smooth function. Then there exists a conformal map $\bar{f}: \bar{\Sigma} \rightarrow \mathbb{S}^{2}$ such that $\int_{\bar{\Sigma}} \bar{f} h \mathrm{~d} A=0$ and $\bar{f}$ has degree less than or equal to $\left[\frac{g+3}{2}\right]$, where $[x]$ denotes the integer part of $x$.

Proof of Theorem E. We analyse each one of the three cases.

(i) Let $\left\{E_{1}, E_{2}, E_{3}\right\}$ a local orthonormal frame on $\Sigma$ such that $E_{1}$ and $E_{2}$ are tangent to $\Sigma$ and $E_{3}$ coincides with the unit normal $N$ to $\Sigma$. Since $\Sigma$ is minimal, the Gauss equation gives that

$$
K_{\Sigma}=\bar{K}\left(E_{1} \wedge E_{2}\right)-\frac{1}{2}\|A\|^{2} .
$$

So,

$$
\begin{aligned}
\operatorname{Ric}(N)+2 K_{\Sigma} & =\bar{K}\left(E_{1}, N\right)+\bar{K}\left(E_{2}, N\right)+\bar{K}\left(E_{1}, E_{2}\right)-\|A\|^{2} \\
& =\operatorname{Ric}\left(E_{1}\right)+\operatorname{Ric}\left(E_{2}\right)-\|A\|^{2} .
\end{aligned}
$$

Now let $h \geq 0$ be the first eighenfunction of problem 3.2. By gluing a disc on each boundary component of $\Sigma$, we may view $\Sigma$ as a compact domain of a closed orientable Riemannian surface $\bar{\Sigma}$. So, Lemma 3.2.4 furnishes a conformal map $\bar{f}: \bar{\Sigma} \rightarrow \mathbb{S}^{2}$ such that $\int_{\bar{\Sigma}} \bar{f} h \mathrm{~d} A=0$ and $\operatorname{deg}(\bar{f}) \leq\left[\frac{g+3}{2}\right]$. Let $f$ denote the restriction of $\bar{f}$ to $\Sigma$. If we write $f=\left(f_{1}, f_{2}, f_{3}\right)$, then we know that each component $f_{i}$ is orthogonal to the first eigenfunction $h$. Since $\Sigma$ has index 1, we have:

$$
Q\left(f_{i}, f_{i}\right)=\int_{\Sigma}\left[\left\|\nabla f_{i}\right\|^{2}-\left(\operatorname{Ric}(N)+\|A\|^{2}\right) f_{i}^{2}\right] \mathrm{d} A-\int_{\partial \Sigma} q f_{i}^{2} \mathrm{~d} L \geq 0 .
$$

Summing over $i$ and since $\sum_{i=1}^{3}\left|f_{i}\right|^{2}=1$, we get

$$
\int_{\Sigma}\left[\|\nabla f\|^{2}-\left(\operatorname{Ric}(N)+\|A\|^{2}\right)\right] \mathrm{d} A-\int_{\partial \Sigma} q \mathrm{~d} L \geq 0 .
$$

By the conformality of $\bar{f}$,

$$
\int_{\Sigma}\|\nabla f\|^{2} \mathrm{~d} A<\int_{\bar{\Sigma}}\|\nabla \bar{f}\|^{2} \mathrm{~d} A=2 \operatorname{Area}(\bar{f}(\bar{\Sigma}))=2 \operatorname{Area}\left(\mathbb{S}^{2}\right) \operatorname{deg}(\bar{f}) \leq 8 \pi\left[\frac{g+3}{2}\right] .
$$

Therefore,

$$
\int_{\Sigma}\left(\operatorname{Ric}(N)+\|A\|^{2}\right) \mathrm{d} A+\int_{\partial \Sigma} q \mathrm{~d} L<8 \pi\left[\frac{g+3}{2}\right]
$$

We now use equation (3.13) and the hypotheses of curvature Ric $\geq 0$ and $H_{\partial M} \geq 0$ to 
continue:

$$
-2 \int_{\Sigma} K_{\Sigma} \mathrm{d} A \leq \int_{\Sigma}\left(\operatorname{Ric}\left(E_{1}\right)+\operatorname{Ric}\left(E_{2}\right)-2 K_{\Sigma}\right) \mathrm{d} A<8 \pi\left[\frac{g+3}{2}\right]-\int_{\partial \Sigma} q \mathrm{~d} L .
$$

By Gauss-Bonnet theorem,

$$
-2\left[2 \pi(2-2 g-k)-\int_{\partial \Sigma} k_{g} \mathrm{~d} L\right]<8 \pi\left[\frac{g+3}{2}\right]-\int_{\partial \Sigma} q \mathrm{~d} L .
$$

Applying Lemma 3.2.1 and rearanging, we arive at

$$
\int_{\partial \Sigma} k_{g} \mathrm{~d} L<2 \pi\left[9-(-1)^{g}-2(g+k)\right]
$$

as we wanted. The particular case mentioned in the theorem follows immediately.

(ii) To prove this item, we use the identity

$$
\operatorname{Ric}(N)=\frac{1}{2}\left(R_{M}+H_{\Sigma}^{2}-\|A\|^{2}\right)-K_{\Sigma} .
$$

Let $f: \Sigma \rightarrow \mathbb{S}^{2}$ be the same function of item (i). As before,

$$
\int_{\Sigma}\left[\|\nabla f\|^{2}-\left(\operatorname{Ric}(N)+\|A\|^{2}\right)\right] \mathrm{d} A-\int_{\partial \Sigma} q \mathrm{~d} L \geq 0 .
$$

Using equation (3.15) and inequality (3.14), we obtain

$$
\frac{1}{2} \int_{\Sigma}\left(R_{M}+\|A\|^{2}\right) \mathrm{d} A-\int_{\Sigma} K_{\Sigma} \mathrm{d} A+\int_{\partial \Sigma} q \mathrm{~d} L<8 \pi\left[\frac{g+3}{2}\right] .
$$

Now we use Gauss-Bonnet therorem and Lemma 3.2.1 to write

$$
\frac{1}{2} \int_{\Sigma}\left(R_{M}+\|A\|^{2}\right) \mathrm{d} A+\frac{1}{\sin \theta} \int_{\partial \Sigma} H_{\partial M} \mathrm{~d} L<8 \pi\left[\frac{g+3}{2}\right]+2 \pi(2-2 g-k) .
$$

Finally, we use the curvature assumptions to get

$$
\frac{1}{2} \inf _{M} R_{M}|\Sigma|+\frac{1}{\sin \theta} \inf _{\partial M} H_{\partial M}|\partial \Sigma|<2 \pi\left[7-(-1)^{g}-k\right],
$$

as we wanted.

(iii) Item (a) follows from Theorem A, since under the curvature hypotheses $\Sigma$ must be a disc. Item (b) follows from from item (ii) of the current theorem (again, $\Sigma$ must be a disc).

Remark 3.2.2. The scalar curvature in [FP14] is one half of ours. This is why a factor $4 \pi$ appears in item (iii) of Theorem E instead of the $2 \pi$ of Theorem 1.2 in [FP14]. 


\section{Chapter 4}

\section{Systolic inequalities}

In this last chapter, which partially contains the text of the article [Lon20], we formally introduce the notion of homological systoles for Riemannian $n$-manifolds with (possibly empty) boundary. After quickly presenting some properties, we prove Theorems F, H and G in Section 4.2. Finally, we discuss in Section 4.3 which smooth 3-manifolds satisfy the hypotheses of these theorems.

\subsection{Homological systoles}

Let us recall the definition of systole mentioned in the Introduction. For a Riemannian manifold $\left(M^{n}, g\right)$ and an integer $1 \leq k<n$, its homological $k$-systole is given by

$$
\operatorname{sys}_{k}(M)=\inf \left\{\mathcal{H}^{k}(\Sigma): \Sigma^{k} \subset M \text { closed and embedded, }[\Sigma] \neq 0 \in H_{k}(M ; \mathbb{Z})\right\},
$$

where $\mathcal{H}^{k}(\cdot)$ denotes the $k$-dimensional Hausdorff measure. If $H_{k}(M ; \mathbb{Z})=0$, we take $\operatorname{sys}_{k}(M)$ to be infinite.

It is also possible to define a homological systole for Riemannian manifolds with boundary. If $\left(M^{n}, g\right)$ is such a manifold and $1 \leq k<n$ is a positive integer, we define its relative homological $k$-systole by

$$
\operatorname{sys}_{k}(M, \partial M)=\inf \left\{\mathcal{H}^{k}(\Sigma): \Sigma^{k} \in \mathcal{S}_{k} \text { and }[\Sigma] \neq 0 \in H_{k}(M, \partial M ; \mathbb{Z})\right\},
$$

where $\mathcal{S}_{k}$ denotes the set of all compact and embedded $k$-dimensional submanifolds $\Sigma^{k} \subset M$ with boundary such that $\Sigma \cap \partial M=\partial \Sigma$. Notice that any surface in $\mathcal{S}_{k}$ that represents a nonzero element of $H_{k}(M, \partial M ; \mathbb{Z})$ must be orientable, since we are working with integer coefficients. If $H_{k}(M, \partial M ; \mathbb{Z})=0$, we take $\operatorname{sys}_{k}(M, \partial M)$ to be infinite.

Finally, if $\left(M^{n}, g\right)$ is a Riemannian manifold with boundary and $1 \leq k<n-1$ is a positive integer, the boundary homology $k$-systole of $\partial M$ is the number

$$
\operatorname{sys}_{k}^{\partial}(\partial M)=\inf \left\{\mathcal{H}^{k}(\partial \Sigma): \Sigma^{k+1} \in \mathcal{S}_{k+1} \text { and }[\partial \Sigma] \neq 0 \in H_{k}(\partial M ; \mathbb{Z})\right\} .
$$

Again, if $H_{k}(\partial M ; \mathbb{Z})=0$ or $H_{k+1}(M, \partial M ; \mathbb{Z})=0$, we convention that $\operatorname{sys}_{k}^{\partial}(\partial M)$ is infinite.

Notice that there is an abuse of notation when we write $\operatorname{sys}_{k}(M), \operatorname{sys}_{k}(M, \partial M)$ and $\operatorname{sys}_{k}^{\partial}(\partial M)$, since these systoles are defined for Riemannian manifolds. However, there will be no risk of confusion since the Riemannian metric will be clear from the context.

Convention. From now on, all homology groups will always be taken with integer coefficients unless otherwise stated.

Let us begin with a simple relation between the systoles of a product $M \times N$ and the systoles of the factors $M$ and $N$. 
Proposition 4.1.1. Let $\left(M^{m}, g_{M}\right)$ and $\left(N^{n}, g_{N}\right)$ be Riemannian manifolds without boundary and endow the product $M \times N$ with the product metric $g_{M} \oplus g_{N}$. If $1 \leq k \leq \min \{m, n\}$, then

$$
\operatorname{sys}_{k}(M \times N) \leq \min \left\{\operatorname{sys}_{k}(M), \operatorname{sys}_{k}(N)\right\},
$$

provided the right hand side is finite. A similar statement holds for manifolds with boundary.

Proof. For $\varepsilon>0$, let $\Sigma^{k} \subset M$ a closed and embedded $k$-submanifold determining a nontrivial class in $H_{k}(M)$ and such that

$$
\operatorname{sys}_{k}(M) \leq \mathcal{H}^{k}(\Sigma)<\operatorname{sys}_{k}(M)+\varepsilon .
$$

Fix a point $y \in N$, let $\iota_{y}: M \rightarrow M \times N$ denote the map $\iota_{y}(x)=(x, y), x \in M$, and denote by $\pi_{M}: M \times N \rightarrow M$ the projection. Since $\pi_{M} \circ \iota_{y}$ is the identity on $M$, the induced homomorphism $\left(\iota_{y}\right)_{*}: H_{k}(M) \rightarrow H_{k}(M \times N)$ is injective. So, $\iota_{y}(\Sigma)=\Sigma \times\{y\}$ is a closed and embedded $k$ submanifold of the product $M \times N$ determining a nontrivial class in $H_{k}(M \times N)$. Thus,

$$
\operatorname{sys}_{k}(M \times N) \leq \mathcal{H}^{k}(\Sigma \times\{y\})=\mathcal{H}^{k}(\Sigma)<\operatorname{sys}_{k}(M)+\varepsilon .
$$

Letting $\varepsilon \rightarrow 0$ shows that $\operatorname{sys}_{k}(M \times N) \leq \operatorname{sys}_{k}(M)$. Analogously for $N$.

The systoles are also well behaved with respect to finite covering maps, according to the next proposition.

Proposition 4.1.2. Let $G$ be a finite group acting properly and discontinuously by isometries on a Riemannian manifold $\left(M^{n}, g\right)$ without boundary, and denote by $\pi: M \rightarrow M / G$ the corresponding Riemannian covering map. Then

$$
\operatorname{sys}_{k}(M) \leq|G| \operatorname{sys}_{k}(M / G) .
$$

provided that both sides are finite. Here, $|G|$ denotes the order of $G$.

Proof. For $\varepsilon>0$, let $\Sigma^{k} \subset M / G$ be a closed, connected and embedded $k$-submanifold determining a nontrivial class in $H_{k}(M / G)$ and such that

$$
\operatorname{sys}_{k}(M) \leq \mathcal{H}^{k}(\Sigma)<\operatorname{sys}_{k}(M)+\varepsilon .
$$

Let $\tilde{\Sigma}=\pi^{-1}(\Sigma) \subset M$. Then $\tilde{\Sigma}$ is closed embedded and determines a nontrivial class in $H_{k}(M)$. Indeed,

$$
\pi_{*}([\tilde{\Sigma}])=[\pi(\tilde{\Sigma})]=[\Sigma] \neq 0 \in H_{k}(M / G) .
$$

So, $\mathcal{H}^{k}(\tilde{\Sigma}) \geq \operatorname{sys}_{k}(M)$. On the other hand, $\mathcal{H}^{k}(\tilde{\Sigma})=|G| \mathcal{H}^{k}(\Sigma)$. Combining all this information, we get

$$
\operatorname{sys}_{k}(M) \leq \mathcal{H}^{k}(\tilde{\Sigma})=|G| \mathcal{H}^{k}(\Sigma)<|G|\left(\operatorname{sys}_{k}(M / G)+\varepsilon\right) .
$$

Letting $\varepsilon \rightarrow 0$ gives the desired result.

Finally, we present a result for systoles in some Riemannian fibre bundles.

Proposition 4.1.3. Let $\pi:\left(M^{n+l}, g_{M}\right) \rightarrow\left(B^{n}, g_{B}\right)$ be a Riemannian submersion with compact fibre $F^{l}$, where $(M, g)$ is a complete Riemannian manifold and $F \cong \pi^{-1}(b) \subset M$ is totally geodesic for every $b \in B$. If $F$ has nonvanishing Euler characteristic, then

$$
\operatorname{sys}_{l+k}(M) \leq \mathcal{H}^{l}(F) \operatorname{sys}_{k}(B),
$$

provided that the systoles are taken with rational coefficients and are finite. If the Euler characteristic of $F$ is \pm 1 , then the same inequality holds with integer coefficients. 
Proof. First notice that, under the hypoteses, $\pi: M \rightarrow B$ is a fibre bundle and all the fibres are isometric to each other (see [Her60] or Lemma 1.4.3 and Theorem 1.4.1 in [GW09]).

Let $\varepsilon>0$ and let $\Sigma^{k} \subset B$ be a closed, connected and embedded $k$-submanifold determining a nontrivial class in $H_{k}(B ; \mathbb{Q})$ and such that

$$
\operatorname{sys}_{k}(B) \leq \mathcal{H}^{k}(\Sigma)<\operatorname{sys}_{k}(B)+\varepsilon .
$$

Then $\tilde{\Sigma}=\pi^{-1}(\Sigma) \subset M$ is a closed and embedded $(k+l)$-submanifold, whose Hausdorff measure is $\mathcal{H}^{k+l}(\tilde{\Sigma})=\mathcal{H}^{l}(F) \mathcal{H}^{k}(\Sigma)$ by the coarea formula.

We now show that $\tilde{\Sigma}$ determines a nontrivial class in $H_{k+l}(M, \mathbb{Q})$. For this, let $\pi^{!}: H^{k+l}(M ; \mathbb{Q}) \rightarrow$ $H^{k}(B ; \mathbb{Q})$ be the map given by integration along the fibres. We have $\langle\alpha,[\tilde{\Sigma}]\rangle=\left\langle\pi^{!}(\alpha),[\Sigma]\right\rangle$, for any $\alpha \in H^{k+l}(M, \mathbb{Q})$, and where $\langle\cdot, \cdot\rangle$ denotes the pairing between homology and cohomology. If we ensure that the right hand side is nonzero, this will show that $[\tilde{\Sigma}]$ is nonzero in homology. For this, let $\gamma \in H^{k}(B ; \mathbb{Q})$ be the dual of $[\Sigma]$, so that $\langle\gamma,[\Sigma]\rangle=1$. Also let $V=\operatorname{ker}(D \pi) \subset T M$ be the vertical bundle and $e(V) \in H^{k}(M ; \mathbb{Q})$ its Euler class. Then, since $\pi^{!}$is a $H^{*}(B)$-module homomorphism, we have

$$
\pi^{!}\left(e(V) \smile \pi^{*}(\gamma)\right)=\pi^{!}(e(V)) \gamma=\chi(F) \gamma
$$

Since $\chi(F) \neq 0$ and we are working with rational coefficients, we can divide by $\chi(F)$ to conclude that $\gamma$ is in the image of $\pi^{\text {! }}$. Then,

$$
\left\langle(\chi(F))^{-1} e(V) \smile \pi^{*}(\gamma),[\tilde{\Sigma}]\right\rangle=\langle\gamma,[\Sigma]\rangle=1,
$$

which proves that $[\tilde{\Sigma}]$ is nontrivial in homology. So, $\mathcal{H}^{k+l}(\tilde{\Sigma}) \geq \operatorname{sys}_{k+l}(M)$ and

$$
\operatorname{sys}_{k+l}(M) \leq \mathcal{H}^{k+l}(\tilde{\Sigma})=\mathcal{H}^{l}(F) \mathcal{H}^{k}(\Sigma)<\mathcal{H}^{l}(F)\left(\operatorname{sys}_{k}(B)+\varepsilon\right)
$$

Letting $\varepsilon \rightarrow 0$ gives the desired result.

When working with integer coefficients, we can no longer divide by $\chi(F)$ in the argument above, unless $\chi(F)= \pm 1$, in which case the proof is the same.

\subsection{Results}

As mentioned in the Introduction, to better understand a certain Riemannian manifold, it is often useful to study its interesting submanifolds. In this vein, many authors have established relations between geometric properties of the manifold (such as curvatures) and geometric properties of some class of special submanifolds. For instance, a classical result due to Toponogov [Top59] states that every simple closed geodesic $\gamma$ on a two-dimensional surface $(\Sigma, g)$ satisfies

$$
\operatorname{Length}(\gamma) \inf _{\Sigma} K \leq 4 \pi^{2}
$$

where $K$ denotes the Gaussian curvature of $\Sigma$. Equality holds if and only if $\Sigma$ is isometric to the unit sphere $\mathbb{S}^{2}$ up to scaling.

In the 3-dimensional setting, Schoen and Yau [SY79] proved that any compact and connected area-minimising surface in a Riemannian 3-manifold $(M, g)$ with positive scalar curvature must be homeomorphic either to $\mathbb{S}^{2}$ or to $\mathbb{R P}^{2}$. When this surface $\Sigma$ is a projective plane, then it is possible to estimate its area from above by

$$
\operatorname{Area}(\Sigma) \inf _{M} R_{M} \leq 12 \pi
$$

see [HBN10a]. Moreover, equality happens if and only if $(M, g)$ is isometric to $\mathbb{R} \mathbb{P}^{3}$ up to scaling. In the same year, Bray, Brendle and Neves analysed the case of area-minimising 2-spheres. Assuming that $\left(M^{3}, g\right)$ is a compact Riemannian 3-manifold with $\pi_{2}(M) \neq 0$, they defined the following 
quantity:

$$
\mathcal{A}(M, g)=\inf \left\{\operatorname{Area}\left(\mathbb{S}^{2}, f^{*} g\right): f \in \mathcal{F}\right\},
$$

where $\mathcal{F}$ is the set of all smooth maps $f: \mathbb{S}^{2} \rightarrow M$ which represent a nontrivial element of $\pi_{2}(M)$. They proved that

$$
\mathcal{A}(M, g) \inf _{M} R_{M} \leq 8 \pi
$$

and if equality holds, then the universal cover of $(M, g)$ is isometric to the standard cylinder $\mathbb{S}^{2} \times \mathbb{R}$ up to scaling.

In a recent paper, Stern [Ste] gave a proof for the following systolic inequality, which is a weaker variant of the theorem proved by Bray, Brendle and Neves described above:

Theorem 4.2.1. On a closed, connected and oriented Riemannian 3-manifold $\left(M^{3}, g\right)$ with positive scalar curvature $R_{M}$ and $H_{2}(M) \neq 0$, we have

$$
\operatorname{sys}_{2}(M) \inf _{M} R_{M} \leq 8 \pi .
$$

Moreover, if equality holds, then the universal cover of $M$ is isometric to the standard cylinder $\mathbb{S}^{2} \times \mathbb{R}$ up to scaling, where $\mathbb{S}^{2}$ is the unit round sphere.

Our first result in this chapter is a version of the previous theorem for 3-manifolds with boundary. The 2-systole is replaced by the relative 2 -systole, and the constant that appears in the right hand side is $4 \pi$, due to the presence of a boundary.

Theorem F. Let $\left(M^{3}, g\right)$ be a compact, connected and oriented Riemannian 3-manifold with nonempty boundary. Assume that $H_{2}(M, \partial M) \neq 0$. If $M$ has positive scalar curvature $\left(R_{M}>0\right)$ and weakly mean-convex boundary $\left(H_{\partial M} \geq 0\right)$, then

$$
\operatorname{sys}_{2}(M, \partial M) \inf _{M} R_{M} \leq 4 \pi .
$$

Moreover, if equality holds, then the universal cover of $M$ is isometric to the cylinder $\mathbb{S}_{+}^{2} \times \mathbb{R}$ up to scaling, where $\mathbb{S}_{+}^{2}$ is a closed hemisphere of the unit round sphere.

Remark 4.2.1. To make sense or the relative 2-systole, we will always assume that $H_{2}(M, \partial M) \neq$ 0 . So, a natural question arises: are there geometric obstructions for $H_{2}(M, \partial M)$ to be nonzero for a given compact, connected and orientable Riemannian 3-manifold $(M, g)$ with nonempty boundary? For instance, Fraser and Li [FL14, Lemma 2.1] showed that $H_{2}(M, \partial M)$ vanishes if the Ricci curvature of $M$ is nonnegative and $\partial M$ is strictly convex. They actually proved a much stronger result: under these geometric assumptions, $M$ is diffeomorphic to the 3 -ball $\mathbb{B}^{3}$ (see Theorem 2.11 in (FL14]). In Section 4.3, we will characterize those smooth 3-manifolds $M$ which admit metrics of positive scalar curvature and mean-convex boundary such that $H_{2}(M, \partial M) \neq 0$ (see Proposition 4.3.2).

For Theorems $\mathrm{F}$ and $\mathrm{G}$, we shall make use of the following result:

Theorem 4.2.2. [BS, Theorem 1.1] Let $\left(M^{3}, g\right)$ be a compact, connected and oriented Riemannian 3-manifold with nonempty boundary. For a harmonic map $u: M \rightarrow \mathbb{S}^{1}=\mathbb{R} / \mathbb{Z}$ satisfying homogeneous Neumann condition, we have the inequality

$$
2 \pi \int_{\mathbb{S}^{1}} \chi\left(\Sigma_{\theta}\right) \geq \int_{\mathbb{S}^{1}}\left(\int_{\Sigma_{\theta}} \frac{1}{2}\left(\|\mathrm{~d} u\|^{-2}\|\operatorname{Hess}(u)\|^{2}+R_{M}\right)+\int_{\partial \Sigma_{\theta}} H_{\partial M}\right)
$$

where $R_{M}$ is the scalar curvature of $M, H_{\partial M}$ is the mean curvature of $\partial M$ with respect to the inward pointing unit normal, $\Sigma_{\theta}=u^{-1}(\theta)$ is a regular level set of $u$ and $\chi(\cdot)$ denotes the Euler characteristic. 
Notice that every regular level set $\Sigma_{\theta}$ of $u$ in Theorem 4.2 .2 meets the boundary of $M$ orthogonally. This will be important in the proof of Theorem F. First, a simple lemma.

Lemma 4.2.3. Let $\left(D^{2}, g\right)$ be a Riemannian 2-disc. If it has positive constant Gaussian curvature and $\partial D$ has constant geodesic curvature, then $D$ is isometric to a closed geodesic disc in the unit sphere $\mathbb{S}^{2}$ up to scaling.

Proof. By the Fundamental Theorem of Isometric Immersions (see Theorem 1.25 in [DT19]), there exists an isometric immersion $f: D \rightarrow \mathbb{S}^{2}$ if we rescale the metric on $D$. Since $\partial D$ has constant geodesic curvature, $C=f(\partial D) \subset \mathbb{S}^{2}$ must be a round circle. It is clear that $f(D)$ must be one of the connected components of $\mathbb{S}^{2} \backslash C$. We now show that $f: D \rightarrow \mathbb{S}^{2}$ is injective, which will complete the proof.

Let $\Sigma$ be the double of $D$, obtained by gluing two copies of $D$ along its boundary via the identity map. Let $j: \Sigma \rightarrow \Sigma$ be the natural reflection map and let $J: \mathbb{S}^{2} \rightarrow \mathbb{S}^{2}$ be the reflection with respect to $C$. Define a map $F: \Sigma \rightarrow \mathbb{S}^{2}$ by

$$
F(x)=J(f(j(x))), \quad x \in \Sigma .
$$

It is easy to show that $F$ is a local homeomorphism. Since $\Sigma$ is compact, $F$ is a covering map. Since $\mathbb{S}^{2}$ is simply connected, $F$ must be a global homeomorphism. In particular, $f$ is injective.

Proof of Theorem F. Recall that Poincaré-Lefschetz duality gives an isomorphism

$$
H_{2}(M, \partial M ; \mathbb{Z}) \cong H^{1}(M ; \mathbb{Z}) \cong\left[M: \mathbb{S}^{1}\right] .
$$

Since we are assuming that $H_{2}(M, \partial M) \neq 0$, there is a nontrivial homotopy class $[v] \in\left[M: \mathbb{S}^{1}\right]$. Applying standard Hodge theory to the cohomology class $v^{*}(\mathrm{~d} \theta) \in H_{\mathrm{dR}}^{1}(M)$ provides an energyminimising representative $u: M \rightarrow \mathbb{S}^{1}$. It can be shown that this function is harmonic and satisfies homogeneous Neumann condition along $\partial M$ (see Chapter 5, Section 9 in [Tay96] or Lemma 1 in [RV95]).

In order to prove the theorem, fix such a map $u: M \rightarrow \mathbb{S}^{1}$. By Theorem 4.2 .2 and by the fact that $H^{\partial M} \geq 0$, we have the following inequalities:

$$
\begin{aligned}
2 \pi \int_{\mathbb{S}^{1}} \chi\left(\Sigma_{\theta}\right) & \geq \int_{\mathbb{S}^{1}}\left(\int_{\Sigma_{\theta}} \frac{1}{2}\left(\|\mathrm{~d} u\|^{-2}\|\operatorname{Hess}(u)\|^{2}+R_{M}\right)+\int_{\partial \Sigma_{\theta}} H^{\partial M}\right) \\
& \geq \frac{1}{2} \inf _{M} R_{M} \int_{\mathbb{S}^{1}} \operatorname{Area}\left(\Sigma_{\theta}\right) .
\end{aligned}
$$

Now notice that, whenever $\Sigma_{\theta}$ is a regular level set of $u$, it holds that every connected component of $\Sigma_{\theta}$ represents a nontrivial class in $H_{2}(M, \partial M)$. Indeed, the gradient 1-form $h=u^{*}(\mathrm{~d} \theta)$ induced by $u$ is harmonic $\left(\mathrm{d} h=\mathrm{d}^{*} h=0\right)$ and satisfies $h(X)=0$, where $X$ is the outward unit normal to $\partial M$. This implies that the Hodge dual $* h$ is closed and satisfies $\iota_{\partial M}^{*}(* h)=0$ on $\partial M$, where $\iota_{\partial M}: \partial M \rightarrow M$ denotes the inclusion. This said, let $S$ be a connected component of $\Sigma_{\theta}$. Then

$$
\int_{S} * h=\int_{S}|h|>0
$$

which implies the claim.

Also observe that, if $N(\theta)$ denotes the number of connected components of $\Sigma_{\theta}$, then $\chi\left(\Sigma_{\theta}\right) \leq$ $N(\theta)$. This holds simply because $\chi(S) \leq 1$ for any compact and connected surface with boundary.

Combining these facts with inequality (4.1), we obtain 


$$
\begin{aligned}
2 \pi \int_{\mathbb{S}^{1}} N(\theta) & \geq 2 \pi \int_{\mathbb{S}^{1}} \chi\left(\Sigma_{\theta}\right) \geq \frac{1}{2} \inf _{M} R_{M} \int_{\mathbb{S}^{1}} \operatorname{Area}\left(\Sigma_{\theta}\right) \\
& \geq \frac{1}{2} \operatorname{sys}_{2}(M, \partial M) \inf _{M} R_{M} \int_{\mathbb{S}^{1}} N(\theta) .
\end{aligned}
$$

Cancelling factors, we get

$$
\operatorname{sys}_{2}(M, \partial M) \inf _{M} R_{M} \leq 4 \pi,
$$

as we wanted.

Suppose now that equality holds. Then, analysing all the previous steps, we have

(i) $\operatorname{Hess}(u) \equiv 0$ on $M$;

(ii) $R_{M} \equiv \inf _{M} R_{M}>0$ is constant along $M$;

(iii) $H_{\partial M} \equiv 0$ along $\partial M$;

(iv) $\chi\left(\Sigma_{\theta}\right)=N(\theta)$ for every $\theta \in \mathbb{S}^{1}$.

Firstly, notice that condition (i) implies that $\mathrm{d} u$ has constant norm (different from 0). So, every level set $\Sigma_{\theta}$ is regular and totally geodesic. Indeed, let $A$ denote the second fundamental form of a level set of $u$, and let $X, Y$ be tangent vectors of that level set. Then

$$
\begin{aligned}
A(X, Y) & =\left\langle\bar{\nabla}_{X} Y, \frac{\nabla u}{\|\nabla u\|}\right\rangle=\frac{1}{\|\nabla u\|}\left\langle\bar{\nabla}_{X} Y, \nabla u\right\rangle=-\frac{1}{\|\nabla u\|}\left\langle\bar{\nabla}_{X} \nabla u, Y\right\rangle \\
& =-\frac{1}{\|\nabla u\|} \operatorname{Hess}(u)(X, Y)=0 .
\end{aligned}
$$

Secondly, the Bochner formula for the (harmonic) gradient 1-form $h=u^{*}(\mathrm{~d} \theta)$ reads

$$
\Delta \frac{1}{2}\|h\|^{2}=\|D h\|^{2}+\operatorname{Ric}(h, h) .
$$

Since $\|h\|=\|\mathrm{d} u\|$ is constant and $D h=\operatorname{Hess}(u) \equiv 0$, we get $\operatorname{Ric}(h, h)=\operatorname{Ric}(\nabla u, \nabla u)=0$. Now, the Gauss equation for a level set $\Sigma_{\theta}$,

$$
\operatorname{Ric}(N, N)=\frac{1}{2}\left(R_{M}-R_{\theta}+H_{\theta}^{2}-\left\|A_{\theta}\right\|^{2}\right),
$$

gives that the sectional curvature of $\Sigma_{\theta}$ is constant and equal to $\frac{1}{2} R_{M}$ (which is itself constant by (ii)). Here, $N=\frac{\nabla u}{\|\nabla u\|}$ denotes the unit normal, $R_{\theta}$ the scalar curvature, $H_{\theta}$ the mean curvature and $A_{\theta}$ the second fundamental form of $\Sigma_{\theta}$. This way, each component of a level set of $u$ is isometric to disc (by condition (iv)) of a round sphere.

We now show that the geodesic curvature of the boundary of such a disc $D$ is zero. For this, let $T$ be a unit vector field which is tangent to the boundary of $D$. Since $u$ satisfies the homogeneous Neumann boundary condition, $\{T(p), N(p)\}$ is an orthonormal basis of $T_{p}(\partial M)$ for every $p \in \partial D$, where $N=\frac{\nabla u}{\|\nabla u\|}$. Now let $X$ be the outward unit normal to $\partial M$. Then the geodesic curvature of $\partial D$ is given by $k_{g}=\left\langle\nabla_{T} X, T\right\rangle$, because $X$ is also the outward unit conormal to $\partial D$ in $D$. So, we have:

$$
0=H_{\partial M}=-k_{g}+\left\langle\nabla_{N} X, N\right\rangle
$$


But as $\langle X, \nabla u\rangle=0$, we can write the second term above as

$$
\begin{aligned}
\left\langle\nabla_{N} X, N\right\rangle & =\frac{1}{\|\nabla u\|^{2}}\left\langle\nabla_{\nabla u} X, \nabla u\right\rangle=-\frac{1}{\|\nabla u\|^{2}}\left\langle\nabla_{\nabla u} \nabla u, X\right\rangle \\
& =-\frac{1}{\|\nabla u\|^{2}} \operatorname{Hess}(u)(\nabla u, X)
\end{aligned}
$$

Since we are assuming that the Hessian of $u$ vanishes, this shows that $k_{g}=0$, as we wanted. By Lemma $4.2 .3, D$ is isometric to a hemisphere of the unit sphere $\mathbb{S}^{2}$ up to scaling.

Finally, fixing a connected component $S$ of a level set of $u$, the gradient flow of $u, \Phi: S \times \mathbb{R} \rightarrow M$,

$$
\frac{\partial \Phi}{\partial t}=\frac{\nabla u}{\|\nabla u\|} \circ \Phi
$$

defines a local isometry. Notice that since the gradient of $u$ is tangent to the boundary (by the Neumann condition), the flow is well defined. So, $\Phi$ is a covering map. This completes the proof of the theorem.

We now move to Theorem G. Recall that $M^{3}$ is called weakly irreducible when every smoothly embedded 2-sphere in the interior of $M$ separates.

Theorem G. Let $\left(M^{3}, g\right)$ be a compact, connected and oriented Riemannian 3-manifold with nonempty boundary. Assume that $M$ is weakly irreducible and that $H_{2}(M, \partial M ; \mathbb{Z}) \neq 0$. If $M$ has positive scalar curvature and weakly mean-convex boundary, then

$$
\frac{1}{2} \operatorname{sys}_{2}(M, \partial M) \inf _{M} R_{M}+\operatorname{sys}_{1}^{\partial}(\partial M) \inf _{\partial M} H_{\partial M} \leq 2 \pi .
$$

Moreover, if equality holds, then the universal cover of $M$ is isometric to the cylinder $\mathbb{B}_{r}^{2} \times \mathbb{R}$ up to scaling, where $\mathbb{B}_{r}^{2}$ is a geodesic ball of radius $r=\cos ^{-1}\left(1-\frac{\operatorname{sys}_{2}(M, \partial M)}{2 \pi}\right)$ of the unit round sphere.

Proof of Theorem G. Fix a nontrivial harmonic map $u: M \rightarrow \mathbb{S}^{1}$ satisfying homogeneous Neumann boundary condition. From the proof of the previous theorem, we know that any component $S$ of a regular level set $\Sigma_{\theta}$ of $u$ represents a nontrivial class in $H_{2}(M, \partial M)$. As will be seen in the next section (Proposition 4.3.6), if $M$ is weakly irreducible and has the form given by Carlotto and $\mathrm{Li}$ classification theorem - which is the case - , then the connecting homomorphism $\partial$ : $H_{2}(M, \partial M) \rightarrow H_{1}(\partial M)$ is injective. It follows that $\partial S$ represents a nontrivial class in $H_{1}(\partial M)$ and thus, Length $(\partial S) \geq \operatorname{sys}_{1}^{\partial}(\partial M)$. So, invoking Theorem 4.2.2 as before, we have

$$
\begin{aligned}
2 \pi \int_{\mathbb{S}^{1}} N(\theta) & \geq 2 \pi \int_{\mathbb{S}^{1}} \chi\left(\Sigma_{\theta}\right) \geq \int_{\mathbb{S}^{1}}\left(\int_{\Sigma_{\theta}} \frac{1}{2}\left(\|\mathrm{~d} u\|^{-2}\|\operatorname{Hess}(u)\|^{2}+R_{M}\right)+\int_{\partial \Sigma_{\theta}} H_{\partial M}\right) \\
& \geq \frac{1}{2} \inf _{M} R_{M} \int_{\mathbb{S}^{1}} \operatorname{Area}\left(\Sigma_{\theta}\right)+\inf _{\partial M} H_{\partial M} \int_{\mathbb{S}^{1}} \operatorname{Length}\left(\partial \Sigma_{\theta}\right) \\
& \geq \frac{1}{2} \operatorname{sys}_{2}(M, \partial M) \inf _{M} R_{M} \int_{\mathbb{S}^{1}} N(\theta)+\operatorname{sys}_{1}^{\partial}(\partial M) \inf _{\partial M} H_{\partial M} \int_{\mathbb{S}^{1}} N(\theta)
\end{aligned}
$$

Cancelling factors, we get

$$
\frac{1}{2} \operatorname{sys}_{2}(M, \partial M) \inf _{M} R_{M}+\operatorname{sys}_{1}^{\partial}(\partial M) \inf _{\partial M} H_{\partial M} \leq 2 \pi,
$$

as we wanted. The analysis of the equality case goes as in the proof of Theorem F. Just notice that, in the present situation, the boundary of $M$ need not be minimal. Therefore, each component of a level set of $u$ is isometric to a geodesic ball of area $\operatorname{sys}_{2}(M, \partial M)$ of the round sphere, as we wanted.

We conclude by proving Theorem $\mathrm{H}$. 
Theorem H. Let $\left(M^{3}, g\right)$ be a compact, connected and oriented Riemannian 3-manifold with nonempty boundary. Assume that $M$ is weakly irreducible and $H_{2}(M, \partial M ; \mathbb{Z}) \neq 0$. If $M$ has positive scalar curvature and weakly mean-convex boundary, then there exists a properly embedded free boundary stable minimal disc $D$ in $M$ such that $[D] \neq 0$ in $H_{2}(M, \partial M ; \mathbb{Z})$ and $\operatorname{Area}(D)=\operatorname{sys}_{2}(M, \partial M)$.

Proof of Theorem H. Let $\left\{\Sigma_{n}\right\}_{n \geq 1}$ be a minimising sequence for $\operatorname{sys}_{2}(M, \partial M)$, that is, each $\Sigma_{n}$ is a compact, oriented and embedded surface determining a nonzero class in $H_{2}(M, \partial M)$, and $\operatorname{Area}\left(\Sigma_{n}\right) \rightarrow \operatorname{sys}_{2}(M, \partial M)$ as $n \rightarrow \infty$. Each $\Sigma_{n}$ can be seen as an integer relative 2 -cycle. We then minimise the mass among all relative integral 2-cycles in the homology class of $\Sigma_{n}$ (see Corollary 9.9 in [FF60], for example). This gives rise to an integral relative cycle $\alpha_{n}$ whose support consists of a smooth, orientable, stable and properly embedded minimal surface $\Sigma_{n}^{*}$ (see the proof of Proposition 5.3 in [WZ], the proof of Proposition 10 in [MR17] and references therein). We are going to show that, under our assumption on the weak irreducibility of $M$, there is at least one component of $\Sigma_{n}^{*}$ which has a nonempty boundary.

Assume $\Sigma_{n}^{*}$ has a closed component $S$. Since $S$ and $M$ are oriented, $S$ must be two-sided. Using 1 as a test function for the second variation of area of this surface, and recalling that the scalar curvature of $M$ is positive, we conclude that this component must be a sphere (see the proof of Theorem 5.1 in [SY79]). By hypothesis, this sphere is separating and thus determines the zero class in $H_{2}(M, \partial M)$. So, if all the components of $\Sigma_{n}^{*}$ were closed, $\Sigma_{n}^{*}$ would represent the trivial class in $H_{2}(M, \partial M)$, contradicting the fact that $\alpha_{n}$ is homologous to $\Sigma_{n}$ and $\left[\Sigma_{n}\right] \neq 0$.

Thus, from what we have just shown and since $\Sigma_{n}^{*}$ is not null-homologous (in relative homology), it must have at least one component with boundary which is also not null-homologous. Call it $D_{n}$. Then $\left\{D_{n}\right\}_{n \geq 1}$ is a sequence of free boundary and properly embedded stable minimal surfaces of uniformly bounded area. By Theorem 1.2 and Theorem 6.1 in [LZ20], there is a subsequence $\left\{D_{n_{k}}\right\}_{k \geq 1}$ that converges smoothly and locally uniformly to a free boundary and properly embedded minimal surface $D$. It is easy to see from the convergence that $[D] \neq 0$. Moreover, since $\operatorname{Area}\left(D_{n}\right) \leq$ $\operatorname{Area}\left(\Sigma_{n}^{*}\right) \leq \operatorname{Area}\left(\Sigma_{n}\right)$, it follows that $\operatorname{Area}(D)=\operatorname{sys}_{2}(M, \partial M)$. In particular, $D$ minimises area in its homology class, which implies that $D$ is stable. Finally, we use Theorem 1.2 (iii) in [FP14] (or Theorem $\mathrm{E}(\mathrm{iii}))$ to conclude that $D$ is a disc.

\subsection{Discussion}

In this final section, we will thoroughly analyse the hypotheses of our previous results and completely classify the smooth 3-manifolds which satisfy them.

Firsly notice that in Theorems F, H and G, we always assume that $\left(M^{3}, g\right)$ has positive scalar curvature and weakly mean-convex boundary. So, by Carlotto and Li classification mentioned in the Introduction of this thesis, such a manifold must be diffeomorphic to a connected sum of the form

$$
P_{\gamma_{1}} \# \cdots \# P_{\gamma_{A}} \# \mathbb{S}^{3} / \Gamma_{1} \# \cdots \# \mathbb{S}^{3} / \Gamma_{B} \#\left(\# \#_{i=1}^{C} \mathbb{S}^{2} \times \mathbb{S}^{1}\right) \backslash\left(\sqcup_{i=1}^{D} B_{i}^{3}\right),
$$

where $P_{\gamma_{i}}, i \leq A$, are genus $\gamma_{i}$ handlebodies; $\Gamma_{i}, i \leq B$, are finite subgroups of $S O(4)$ acting freely on $\mathbb{S}^{3} ; B_{i}^{3}, i \leq D$, are disjoint 3-balls in the interior.

Another common hypothesis is that $H_{2}(M, \partial M) \neq 0$. So, will compute this homology group for the 3-manifolds of the form above.

Lemma 4.3.1. Let $M^{3}$ and $N^{3}$ be compact, connected and oriented 3-manifolds, where both may have nonempty boundary. Then

$$
H_{1}(M \# N) \cong H_{1}(M) \oplus H_{1}(N) \text {. }
$$

Proof. When forming the connected sum, we delete small open 3-balls from the interior of $M$ and $N$ and then glue the resulting manifolds along their boundaries via a homeomorphism. Let $S$ denote the resulting embedded 2 -sphere in $M \# N$. We will analyse the long exact sequence of the 
pair $(M \# N, S)$. First notice that this is a good pair, which means that $S$ is closed and admits a neighbourhood $U \supset S$ in $M$ such that $S$ is a strong deformation retract in $U$. Thus,

$$
H_{1}(M \# N, S) \cong H_{1}((M \# N) / S) \cong H_{1}(M \vee N) \cong H_{1}(M) \oplus H_{1}(N)
$$

The long exact sequence of the pair takes the simple form

$$
0 \longrightarrow H_{1}(M \# N) \longrightarrow H_{1}(M \vee N) \longrightarrow 0
$$

which shows that $H_{1}(M \# N) \cong H_{1}(M) \oplus H_{1}(N)$, as we wanted.

Proposition 4.3.2. Let $M^{3}$ be a smooth 3-manifold of the form given by (4.2). Then $H_{2}(M, \partial M) \neq$ 0 if and only if $\gamma_{i} \geq 1$ for some $1 \leq i \leq A$ or $C \neq 0$.

Proof. First notice that

(i) $H_{1}\left(P_{\gamma_{i}}\right)=\mathbb{Z}^{\gamma_{i}}$, since $P_{\gamma_{i}}$ is homotopically equivalent to a bouquet of $\gamma_{i}$ circles.

(ii) $H_{1}\left(\mathbb{S}^{3} / \Gamma_{i}\right) \cong\left(\Gamma_{i}\right)_{\mathrm{ab}}$, where $(\cdot)_{\mathrm{ab}}$ denotes the abelianisation. This is because $H_{1}\left(\mathbb{S}^{3} / \Gamma_{i}\right) \cong$ $\left(\pi_{1}\right)_{\mathrm{ab}}\left(\mathbb{S}^{3} / \Gamma_{i}\right)$ and $\pi_{1}\left(\mathbb{S}^{3} / \Gamma_{i}\right) \cong \Gamma_{i}$.

(iii) $H_{1}\left(\mathbb{S}^{2} \times \mathbb{S}^{1}\right)=\mathbb{Z}$ by Künneth Theorem.

So, by Lemma 4.3.1, we know that $H_{1}(M) \cong \mathbb{Z}^{\gamma+C} \oplus \Gamma$, where $\gamma=\sum_{i=1}^{A} \gamma_{i}$ and $\Gamma$ is a finite group (deleting the open 3-balls does not afect the fundamental group of a 3-manifold, nor $H_{1}$ ). By Poincaré-Lefschetz duality and the Universal Coefficient Theorem, we have

$$
H_{2}(M, \partial M) \cong H^{1}(M) \cong \operatorname{Hom}\left(H_{1}(M), \mathbb{Z}\right) \cong \mathbb{Z}^{\gamma+C} .
$$

This is nonzero exactly when $\gamma \geq 1$ or $C \neq 0$, as desired.

Let us check the topological hypothesis of Theorem H. We thank Joshua Howie for providing the argument.

Proposition 4.3.3. Let $M^{3}$ be a smooth 3-manifold of the form given by (4.2). Then $M$ is weakly irreducible if and only if $C=0$.

Proof. Suppose that $C=0$ and let $\Sigma$ be a smoothly embedded 2-sphere in the interior of $M$. Let $\mathcal{S}=\left\{S_{j}\right\}_{j=1}^{k}$ be a collection of embedded 2-spheres which decompose $M$ into prime summands (see [Kne29] for existence and [Mil62] for uniqueness of the prime decomposition). Look at the intersection of $\Sigma$ with the spheres of $\mathcal{S}$ and let $\Delta$ be an innermost disc on some $S_{k}$. We can surger $\Sigma$ along $\Delta$, which will decompose $\Sigma$ into two embedded 2-spheres (see Figure 4.1). Repeating this process until there are no intersections with $\mathcal{S}, \Sigma$ is decomposed into a collection of embedded 2 -spheres which we call $\Sigma^{\prime}$. Then $\Sigma$ will be nonseparating in $M$ if and only if some component of $\Sigma^{\prime}$ is nonseparating in $M$.

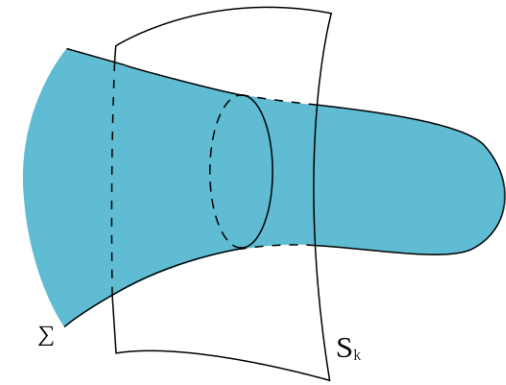

(a) The sphere $\Sigma$ before the surgery.

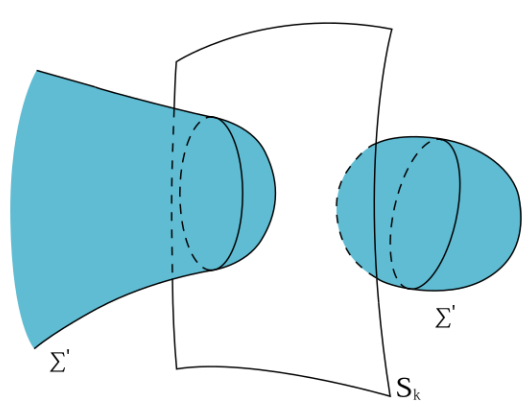

(b) After the surgery, $\Sigma$ is decomposed into two spheres.

Figure 4.1: Two minimal surfaces with the same boundary. 
Each component of $\Sigma^{\prime}$ is contained within a single prime summand of $M$. We then cut $M$ along $\left\{S_{j}\right\}_{j=1}^{k}$, and glue 3-balls onto each 2-sphere boundary component of the resulting 3-manifolds. It is well-known that handlebodies and spherical 3-manifolds are irreducible, which means that every embedded 2-sphere bounds a 3-ball (since the second homology of a handlebody is zero and since any 3-manifold covered by an irreducible 3-manifold is itself irreducible). Thus each component of $\Sigma^{\prime}$ is separating in its respective prime summand and hence in $M$. Removing the $D$ 3-balls from $M$ which are disjoint from $\Sigma$ and $\Sigma^{\prime}$ does not affect whether $\Sigma$ is separating. Therefore $\Sigma$ is separating in $M$.

Conversely, if $C \neq 0$, then we can find a nonseparating 2-sphere $\Sigma_{0}$ in some $\mathbb{S}^{2} \times \mathbb{S}^{1}$ component which is disjoint from each $B_{i}$ and each $S_{j}$. Furthermore, there exists a closed simple curve in $\mathbb{S}^{2} \times \mathbb{S}^{1}$ which intersects $\Sigma_{0}$ transversely exactly once. Isotoping this curve to be disjoint from the balls $B_{i}$ and the spheres $S_{j}$ yields another curve, now in $M$, with the same property. Therefore $\Sigma_{0}$ is nonseparating in $M$.

Finally, we will show that a manifold $M$ of the form given by (4.2) is weakly irreducible if and only if the connecting homomorphism $\partial: H_{2}(M, \partial M) \rightarrow H_{1}(\partial M)$ is injective. We need some auxiliary lemmas.

Lemma 4.3.4. Let $M^{3}$ and $N^{3}$ be compact, connected and oriented 3-manifolds, where both may have nonempty boundary. If at most one manifold has nonempty boundary, then

$$
H_{2}(M \# N) \cong H_{2}(M) \oplus H_{2}(N) .
$$

If both manifolds have nonempty boundary, then

$$
H_{2}(M \# N) \cong H_{2}(M) \oplus H_{2}(N) \oplus \mathbb{Z}
$$

Proof. First assume that both $M$ and $N$ are closed. As in Lemma 4.3.1, we will analyse the long exact sequence of the pair $(M \# N, S)$ :

$$
0 \longrightarrow H_{3}(M \# N) \longrightarrow H_{3}(M \vee N) \longrightarrow H_{2}(S) \longrightarrow H_{2}(M \# N) \longrightarrow H_{2}(M \vee N) \longrightarrow 0
$$

Identifying the groups, the above sequence takes the form

$$
0 \longrightarrow \mathbb{Z} \longrightarrow \mathbb{Z} \oplus \mathbb{Z} \stackrel{\varphi}{\longrightarrow} \mathbb{Z} \stackrel{\psi}{\longrightarrow} H_{2}(M \# N) \longrightarrow H_{2}(M \vee N) \longrightarrow 0
$$

The map $\varphi$ is an epimorphism, since it takes the fundamental class of $M$ minus the 3-ball we delete to perform the connected sum operation to the fundamental class of $S$, up to sign. So, $\psi=0$ and thus, $H_{2}(M \# N)$ is isomorphic to $H_{2}(M \vee N)$, as we wanted.

Now suppose $N$ has nonempty boundary. Then $H_{3}(M \# N)=0$ and $H_{3}(M \vee N) \cong \mathbb{Z}$. So, the long exact sequence above takes the form

$$
0 \longrightarrow \mathbb{Z} \stackrel{\varphi}{\longrightarrow} \mathbb{Z} \stackrel{\psi}{\longrightarrow} H_{2}(M \# N) \longrightarrow H_{2}(M \vee N) \longrightarrow 0
$$

In this situation, $\varphi$ is an isomorphism by the same reason as before. So $\psi=0$ and $H_{2}(M \# N)$ is isomorphic to $H_{2}(M \vee N)$ in this case too.

Finally, let $M$ and $N$ have nonempty boundary. Then $H_{3}(M \# N)=0$ and $H_{3}(M \vee N)=0$. Then the long exact sequence of the pair $(M \# N, S)$ is a short exact sequence of the form

$$
0 \longrightarrow \mathbb{Z} \longrightarrow H_{2}(M \# N) \longrightarrow H_{2}(M \vee N) \longrightarrow 0
$$


By Poincaré-Lefschetz duality and the Universal Coefficient Theorem, we have

$$
H_{2}(M) \cong H^{1}(M, \partial M) \cong \operatorname{Hom}\left(H_{1}(M, \partial M), \mathbb{Z}\right),
$$

and analogously for $H_{2}(N)$. This shows that $H_{2}(M \vee N)$ is free abelian, so the above sequence splits and we get the desired result.

Lemma 4.3.5. Let $M$ be a smooth 3-manifold of the form given by (4.2). Then

$$
H_{2}(M) \cong \mathbb{Z}^{A+C+D-1}
$$

Proof. First notice that

(i) $H_{2}\left(P_{\gamma_{i}}\right)=0$, since $P_{\gamma_{i}}$ is homotopically equivalent to a 1-dimensional CW complex for $\gamma_{i} \geq 1$, and $P_{0}$ is a 3 -ball.

(ii) $H_{2}\left(\mathbb{S}^{3} / \Gamma_{i}\right)=0$, since

$$
H_{2}\left(\mathbb{S}^{3} / \Gamma_{i}\right) \cong H^{1}\left(\mathbb{S}^{3} / \Gamma_{i}\right) \cong \operatorname{Hom}\left(H_{1}\left(\mathbb{S}^{3} / \Gamma_{i}\right), \mathbb{Z}\right)=0
$$

The first isomorphism is just Poincaré duality and the second comes from the Universal Coefficient Theorem. The third group is zero because $H_{1}\left(\mathbb{S}^{3} / \Gamma_{i}\right)$ is the abelianisation of $\Gamma_{i}$, which is finite.

(iii) $H_{2}\left(\mathbb{S}^{2} \times \mathbb{S}^{1}\right)=\mathbb{Z}$ by Künneth Theorem.

Let $N$ be the manifold

$$
P_{\gamma_{1}} \# \cdots \# P_{\gamma_{A}} \# \mathbb{S}^{3} / \Gamma_{1} \# \cdots \# \mathbb{S}^{3} / \Gamma_{B} \#\left(\# \#_{i=1}^{C} \mathbb{S}^{2} \times \mathbb{S}^{1}\right)
$$

so that we can view $M$ as a subset of $N$. Then $H_{2}(N) \cong \mathbb{Z}^{A+C-1}$, by the above considerations and by Lemma 4.3.4.

Suppose that $A \neq 0$. We use a Mayer-Vietoris argument to the open covering $\{U, V\}$ of $N$, where $U=B_{1}^{\prime} \cup \cdots B_{D}^{\prime}$ is a union of slightly larger open balls $B_{i}^{\prime} \supset B_{i}$, and $V=\operatorname{int} M$. We have the following short exact sequence, since $H_{3}(N)=0$ and $H_{1}(U \cap V)=0$ :

$$
0 \longrightarrow H_{2}(U \cap V) \longrightarrow H_{2}(U) \oplus H_{2}(V) \longrightarrow H_{2}(N) \longrightarrow 0
$$

Notice that $H_{2}(U)=0$ and $H_{2}(U \cap V) \cong \mathbb{Z}^{D}$. So, since $H_{2}(N)$ is free abelian, the sequence splits and we have

$$
H_{2}(M) \cong \mathbb{Z}^{D} \oplus H_{2}(N) \cong \mathbb{Z}^{A+C+D-1}
$$

as we wanted.

Now suppose that $A=0$. Then $H_{3}(N) \cong \mathbb{Z}$, since $N$ is closed, connected and orientable. Using the same covering as before, we have the exact sequence

$$
0 \longrightarrow H_{3}(N) \stackrel{h}{\longrightarrow} H_{2}(U \cap V) \longrightarrow H_{2}(U) \oplus H_{2}(V) \longrightarrow H_{2}(N) \longrightarrow 0
$$

Identifying the first two groups, the map $h: \mathbb{Z} \rightarrow \mathbb{Z}^{D}$ is given by $h(n)=(n, \ldots, n)$. So, since $H_{2}(N)$ is free abelian, we have

$$
H_{2}(M) \cong H_{2}(N) \oplus \operatorname{coker}(h) \cong \mathbb{Z}^{C+D-1} .
$$

This completes the proof.

We conclude with the promised result. 
Proposition 4.3.6. Let $M^{3}$ be a smooth 3-manifold with boundary of the form given by (4.2). Then $M$ is weakly irreducible if and only if the connecting homomorphism $\partial: H_{2}(M, \partial M) \rightarrow H_{1}(\partial M)$ is injective.

Proof. Consider the long exact sequence in homology of the pair $(M, \partial M)$ :

$$
0 \longrightarrow H_{3}(M, \partial M) \stackrel{h}{\longrightarrow} H_{2}(\partial M) \longrightarrow H_{2}(M) \longrightarrow H_{2}(M, \partial M) \stackrel{\partial}{\longrightarrow} H_{1}(\partial M) \longrightarrow 0
$$

We know that $H_{3}(M, \partial M)=\mathbb{Z}$ and $H_{2}(\partial M) \cong \mathbb{Z}^{k}$, where $k=A+D$ is the number of connected components of $\partial M$. Putting this information in the above sequence and shortening it, we obtain

$$
0 \longrightarrow \operatorname{coker}(h) \longrightarrow H_{2}(M) \longrightarrow \operatorname{ker}(\partial) \longrightarrow 0
$$

Now notice that $\operatorname{ker}(\partial) \subseteq H_{2}(M, \partial M)$ is free abelian. In fact, $H_{2}(M, \partial M)$ is free abelian, since it is isomorphic to $\operatorname{Hom}\left(H_{1}(M), \mathbb{Z}\right)$. So, this short exact sequence splits and $H_{2}(M) \cong \operatorname{coker}(h) \oplus$ $\operatorname{ker}(\partial)$. But $h$ is given by $h(n)=(n, \ldots, n) \in \mathbb{Z}^{k}$. This way, $H_{2}(M) \cong \mathbb{Z}^{k-1} \oplus \operatorname{ker}(\partial)$. Thus, $\partial: H_{2}(M, \partial M) \rightarrow H_{1}(\partial M)$ is injective precisely when $H_{2}(M) \cong \mathbb{Z}^{k-1}$.

Let us now analyse the concrete case. By Lemma 4.3.5, the second homology group of the manifold under consideration is isomorphic to $\mathbb{Z}^{A+C+D-1}$. So, for this group to be isomorphic to $\mathbb{Z}^{k-1}=\mathbb{Z}^{A+D-1}$, it is necessary and sufficient that $C=0$. Now just apply Proposition 4.3 .3 to conclude the argument. 


\section{References}

[Amb15] L. Ambrozio. Rigidity of area-minimizing free boundary surfaces in mean convex threemanifolds. J. Geom. Anal., 25:1001-1017, 2015. 2, 3, 7, 18, 24

[AS16] A. Ainouz and R. Souam. Stable Capillary Hypersurfaces in a Half-Space or a Slab. Indiana Univ. Math. J., 65(3):813-831, 2016. 13, 15

[Ber] M. Berger. Systoles et applications selon Gromov. Exposé 771, Séminaire N. Bourbaki, Astérisque, 216:279-310. Société Mathématique de France, 1993. 5

[Ber03] M. Berger. A Panoramic View of Riemannian Geometry. Springer-Verlag Berlin Heidelberg, 2003. 5

[Ber08] M. Berger. What is a... Systole? Notices Am. Math. Soc., 55(3):374-376, 2008. 5

[BS] H. Bray and D. Stern. Scalar curvature and harmonic one-forms on three-manifolds with boundary. Available at arXiv:1911.06803v1 [math.DG]. 30

[CK16] J. Choe and M. Koiso. Stable capillary hypersurfaces in a wedge. Pacific J. Math., 280(1):1-15, 2016. 13

[CL] A. Carlotto and C. Li. Constrained deformations of positive scalar curvature metrics. Available at arXiv:1903.11772v2 [math.DG]. 6

[Cou38] R. Courant. The Existence of a Minimal Surface of Least Area Bounded by Prescribed Jordan Arcs and Prescribed Surfaces. Proc. Natl. Acad. Sci. USA, 24(2):97-101, 1938. 2

[Cou50] R. Courant. Dirichlet's Principle, Conformal Mapping, and Minimal Surfaces. SpringerVerlag New York, 1950. 2

[CS18a] L. Ambrozio, A. Carlotto and B. Sharp. Compactness analysis for free boundary minimal hypersurfaces. Calc. Var. PDE, 2018(1):1-39, 2018. 2

[CS18b] L. Ambrozio, A. Carlotto and B. Sharp. Index estimates for free boundary minimal hypersurfaces. Math. Ann., 370(3-4):1063-1078, 2018. 2

[DT19] M. Dajczer and R. Tojeiro. Submanifold Theory: Beyond an Introduction. Universitext. Springer US, 2019. 31

[FF60] H. Federer and W. Fleming. Normal and integral currents. Ann. of Math., 72(2):458-520, 1960. 34

[Fin86] R. Finn. Equilibrium Capillary Surfaces, volume 284 of Grundlehren der mathematischen Wissenschaften. Springer-Verlag New York, first edition, 1986. 13

[FL14] A. Fraser and M. Li. Compactness of the space of embedded minimal surfaces with free boundary in three-manifolds with nonnegative Ricci curvature and convex boundary. $J$. Differ. Geom., 96:183-200, 2014. 30 
[FP14] J. Chen, A. Fraser and C. Pang. Minimal immersions of compact bordered Riemann surfaces with free boundary. Trans. Amer. Math. Soc., 367(4):2487-2507, 2014. 2, 4, 16, $25,26,34$

[Gro83] M. Gromov. Filling Riemannian manifolds. J. Differ. Geom., 18:1-147, 1983. 5

[GW09] D. Gromoll and G. Walschap. Metric Foliations and Curvature, volume 268 of Progress in Mathematics. Birkhäuser Basel, 2009. 29

[HBN10a] M. Eichmair H. Bray, S. Brendle and A. Neves. Area-minimizing projective planes in three-manifolds. Comm. Pure Appl. Math., 63:1237-1277, 2010. 29

[HBN10b] S. Brendle H. Bray and A. Neves. Rigidity of area-minimizing two-spheres in threemanifolds. Commun. Anal. Geom., 18(4):821-830, 2010. 6, 7

[Her60] R. Hermann. A sufficient condition that a mapping of Riemannian manifolds be a fibre bundle. Proc. Amer. Math. Soc., 11:236-242, 1960. 29

[JLBE88] M. do Carmo J. L. Barbosa and J. Eschenburg. Stability of hypersurfaces of constant mean curvature in Riemannian manifolds. Math. Z., (197):123-128, 1988. 14

[Kne29] H. Kneser. Geschlossen Flachen in dreidimensionalen Mannigfaltigkeiten. Jahresber. Deutsch. Math.-Verein., 38:248-260, 1929. 35

[Lí7] R. López. Stability and bifurcation of a capillary surface on a cylinder. SIAM J. Appl. Math., 77(1):108-127, 2017. 13

[Lag60] J. L. Lagrange. Essai d'une nouvelle méthode pour déterminer les maxima et les minima des formules intégrales indéfinies. Miscellanea Taurinensia, 2(1):173-199, 1760. 1

[Li20] C. Li. A polyhedron comparison theorem for 3-manifolds with positive scalar curvature. Invent. Math., 219:1-37, 2020. 18

[Lon20] E. Longa. Sharp Systolic Inequalities for 3-Manifolds with Boundary. J. Geom. Anal., 2020. https://doi.org/10.1007/s12220-020-00548-2. 5, 27

[LZ20] Q. Guang, M. Li and X. Zhou. Curvature estimates for stable free boundary minimal hypersurfaces. J. Reine Angew. Math., 759:245-264, 2020. 34

[Meu85] J. B. Meusnier. Mémoire sur la courbure des surfaces. Mém. Mathém. Phys. Acad. Sci. Paris, prés. par div. Savans, 10:477-510, 1785. Presented in 1776. 1

[Mil62] J. Milnor. A Unique Decomposition Theorem for 3-Manifolds. Amer. J. Math., 84(1):17, 1962. 35

[MR17] L. Mazet and H. Rosenberg. Minimal hypersurfaces of least area. J. Differ. Geom., 106(2):283-316, 2017. 34

[Nit85] J. C. C. Nitsche. Stationary partitioning of convex bodies. Arch. Rational Mech. Anal., 89:1-19, 1985. 13

[Par05] S. H. Park. Every ring type spanner in a wedge is spherical. Math. Ann., 332(3):475-482, 2005. 13

[Pu52] P. M. Pu. Some inequalities in certain nonorientable Riemannian manifolds. Pacific J. Math., 2(1):55-71, 1952. 5

[RR04] M. Ritoré and C. Rosales. Existence and characterization of regions minimizing perimeter under a volume constraint inside euclidean cones. Trans. Am. Math. Soc., 356(11):46014622, 2004. 13 
[RS97] A. Ros and R. Souam. On stability of capillary surfaces in a ball. Pacific J. Math., 178(2), 1997. 13, 15

[RV95] A. Ros and E. Vergasta. Stability for hypersurfaces of constant mean curvature with free boundary. Geom. Dedicata, 56:19-33, 1995. 31

[Sch06] R. Schoen. Minimal submanifolds in higher codimension. Mat. Contemp., 30:169-199, 2006. 16

[Shi56] M. Shiffman. On Surfaces of Stationary Area Bounded by Two Circles, or Convex Curves, in Parallel Planes. Ann. Math., 63(1):77-90, Jan. 1956. 1

[Spi99] M. Spivak. A Comprehensive Introduction to Differential Geometry, volume 4. Publish Or Perish, $3^{\text {rd }}$ edition, 1999. 14

[Ste] D. Stern. Scalar curvature and harmonic maps to $S^{1}$. Available at arXiv:1908.09754v2 [math.DG]. To appear in J. Diff. Geom. 6, 7, 30

[SY79] R. Schoen and S. T. Yau. Existence of incompressible minimal surfaces and the topology of three-dimensional manifolds with nonnegative scalar curvature. Ann. Math., 110(1):127-142, 1979. 29, 34

[Tay96] M. Taylor. Partial differential equations, I-Basic theory, volume 23 of Texts in Applied Mathematics. Springer-Verlag, New York, 1996. 31

[Top59] V. Toponogov. Evaluation of the length of a closed geodesic on a convex surface. Dokl. Akad. Nauk, 124:282-284, 1959. 29

[WX19] G. Wang and C. Xia. Uniqueness of stable capillary hypersurfaces in a ball. Math. Ann., 374:1845-1882, 2019. 13

[WZ] Q. Guang, Z. Wang and X. Zhou. Free boundary minimal hypersurfaces with the least area. Available at arXiv:1801.07036 [math.DG]. 34 